\title{
Reconstruction of the spatial dependency of dielectric and geometrical properties of adhesively bonded structures.
}

\author{
Cameron Mackay ${ }^{\dagger}$, David Hayward ${ }^{\ddagger}$, Anthony J. Mulholland ${ }^{\dagger}$, Sean McKee ${ }^{\dagger}$ \\ and Richard A. Pethrick ${ }^{\ddagger}$ \\ $\dagger$ Department of Mathematics, University of Strathclyde, Glasgow, U.K. \\ $\ddagger$ Department of Pure and Applied Chemistry, University of Strathclyde, \\ Glasgow, U.K.
}

\begin{abstract}
An inverse problem motivated by the nondestructive testing (NDT) of adhesively bonded structures used in the aircraft industry is studied. Using transmission line theory, a model is developed which, when supplied with electrical and geometrical parameters, accurately predicts the reflection coefficient associated with such structures. Particular attention is paid to modelling the connection between the structures and the equipment used to measure the reflection coefficient. The inverse problem is then studied and an optimisation approach employed to recover these electrical and geometrical parameters from experimentally obtained data. In particular the approach focuses on the recovery of spatially varying geometrical parameters as this is paramount to the successful reconstruction of electrical parameters. Reconstructions of structure geometry using this method are found to be in close agreement with experimental observations.
\end{abstract}

\section{Introduction}

An increase in the already wide use of adhesively bonded structures in the aerospace industry has generated significant interest in the nondestructive testing (NDT) of their durability. Deterioration of these structures can arise not only from instant damage such as bird impacts and lightning strikes but also from gradual damage due to water ingress, corrosion and thermal stress. This paper is concerned with the detection of bond deterioration through the use of 
electrical measurements. In particular it explores the role of reflection coefficients in reconstructing the spatial variation of the structural parameters. We develop a forward model and use optimisation to recover the spatial variation in geometrical properties of such structures from experimentally obtained reflection coefficients.

The reflection coefficients can be generated for a range of frequencies between $100 \mathrm{KHz}$ and $6 \mathrm{GHz}$. Water in its liquid state gives rise to dielectric effects at $18 \mathrm{GHz}$ and is therefore undetectable by the reflection coefficient techniques. However it has been established that after some time, the water ingress facilitates the formation of hydroxide bonds in the adhesive layer; these hydroxide bonds may be detected at lower frequencies. Correlation between the average dielectric properties, environmental ageing, water permeation and mechanical properties have been reported $[1,3,2,4,9]$. However, inaccuracies in the dielectric recovery have been encountered for high frequencies and in particular for frequencies at which electrical effects due to the mechanically fatal hydroxide bond would be observed. Investigation into how a spatially varying the geometry of the structure affects the recoverability of dielectric properties has revealed the same inaccuracies to be present, [13]. Thus any errors in the high frequency dielectric recovery can be attributed to the use of a model which has deficient geometrical information. In this NDT application the thickness of the bonded layer is spatially varying and difficult to measure with any precision. Therefore in order to overcome high frequency inaccuracies (and detect hydroxide bonding) the recovery of the geometrical properties will have to be performed simultaneously with the dielectric recovery. In this paper a multilayered media approach is adopted and an adaption of a nonlinear ordinary differential equation, introduced in [16], is solved to yield a Riccati difference equation for the reflection coefficient. An optimisation method is used to reconstruct both electrical parameters and spatially varying geometries from computer simulated and experimental data. Reconstructions of the spatially varying geometry prove to be particularly good. The optimisation method is aided by the derivation of an analytical Jacobian.

\subsection{An Analytical Solution For the Reflection Coefficient in Multilayered Media}

The starting point for this work is that of He and Norgren $[15,17,16]$. However, in contrast to their work, the differential equation relating the reflection coefficient to the physical parameters of the structure and the frequency is solved analytically. This helps us to analyse the uniqueness of the recovered solution and to derive an analytic Jacobian to speed up the optimisation search algorithm. Furthermore, following [14], a multilayered media approach is employed in order to cope with the geometry and other parameters that vary along the 
transmission line. In practice the aluminium plates bounding the dielectric slab are not infinitely conducting: they are, however, still highly conducting. This results in slight penetration of the field into the conductor which contributes to the attenuation of any electromagnetic wave propagating between the two aluminium conductors.

We start by providing a brief outline of the forward model. Transmission line theory exploits a particular set of travelling wave solutions of Maxwell's equations. From these solutions the telegraph equations may be derived in matrix form as [20]

$$
\frac{d}{d z}\left[\begin{array}{l}
V \\
I
\end{array}\right]=\left[\begin{array}{cc}
0 & -j \frac{\omega}{g} \mu \\
-j \omega g \epsilon & 0
\end{array}\right]\left[\begin{array}{c}
V \\
I
\end{array}\right]
$$

where $\epsilon$ is the permittivity, $\mu$ is the permeability, $\omega$ is the frequency, $W$ is the plate width, $d$ is the plate separation, $g=W / d$, and $V(z)$ and $I(z)$ are the voltage between, and current through the plates. In contrast to Norgen and He [16] this expression contains two complex quantities $\epsilon$ and $\mu$ rather than the four real quantities $R, L, C$ and $G$ which characterise the analogous circuit.

The reflection coefficient is measured at either end of the aluminium structure $(z=0$ and $z=L$ for length $L)$ giving two reflection coefficients over a range of frequencies. To utilise this data, a model is derived in a transmission line setting that takes into account loss through both polarisation and the skin effect. The equation for relative permittivity, $\epsilon_{r}$, best describing the dielectric permittivity and the dielectric loss of an epoxy resin adhesive, is the Havriliak-Negami (HN) equation $[7,13,9]$

$$
\epsilon_{r}=\epsilon^{\infty}+\frac{\epsilon^{s}-\epsilon^{\infty}}{\left(1+(j \omega \tau)^{\alpha}\right)^{\beta}}
$$

where $\epsilon^{\infty}$ and $\epsilon^{s}$ are the infinite and static permittivities respectively. The parameter $\alpha$ can be thought of as a measure of the spread of the dielectric relaxation times (in logarithmic scaling). The parameter $\beta$ changes the overall shape of the curves, in essence breaking the symmetry, while the parameter $\tau$ can be interpreted as the mean dielectric relaxation time $[6,5]$.

The effective permeability for a finite value of the resistivity of the conductor is given by [19]

$$
\mu=\mu_{0}+\frac{(1-j)}{d} \pi \sqrt{\frac{2 \mu_{0} \rho}{\omega}} .
$$

where $\mu_{0}$ is the permeability of free space and $\rho$ is the resistivity. The R,L,C and $\mathrm{G}$ description used by Norgren and He [16] results in an ordinary differential equation for the reflection coefficient. Using the HN expression (2) for the permittivity and expression (3) for the permeability, a similar analysis was performed as in [16] to yield a differential equation for the reflection coefficient $r$

$$
\frac{d}{d z} r=2 a r+b\left(1+r^{2}\right)
$$


where $z \in[0, L]$ and

$$
\begin{aligned}
& a=\frac{1}{2} j \omega\left(\epsilon g Z_{0}+\frac{\mu}{g Z_{0}}\right) \\
& b=\frac{1}{2} j \omega\left(\epsilon g Z_{0}-\frac{\mu}{g Z_{0}}\right) .
\end{aligned}
$$

The parameter $Z_{0}$ is the characteristic impedance of the measuring system, normally $50 \Omega$. This equation was obtained through the wave-splitting approach

$$
\left[\begin{array}{l}
V^{+} \\
V^{-}
\end{array}\right]=\frac{1}{2}\left[\begin{array}{cc}
1 & Z_{0} \\
1 & -Z_{0}
\end{array}\right]\left[\begin{array}{l}
V \\
I
\end{array}\right]
$$

where $V^{+}$and $V^{-}$are the right and left travelling voltage waves respectively. These voltages are linked to the reflection coefficient $r(z)$ by

$$
V^{-}(z)=r(z) V^{+}(z)
$$

where

$$
r(z)=\frac{Z(z)-Z_{0}}{Z(z)+Z_{0}}
$$

and $Z(z)$ denotes the impedance of the line at $z$. At the load end of the transmission line, $z=L$, the transmission line is terminated with a load or termination impedance. This load impedance is defined as

$$
Z_{L}=\left.\frac{V(z)}{I(z)}\right|_{z=L} .
$$

Setting $z=L$ in (8) yields the boundary condition

$$
r(L)=\frac{Z_{L}-Z_{0}}{Z_{L}+Z_{0}}
$$

We now depart from the approach of [16] by modelling the spatial variations in the geometry and dielectric properties of the structure using a piecewise constant, multi-layer approach. To obtain the reflection coefficient $r_{0}:=\left.r(z)\right|_{z=0}$, the boundary condition at $z=L$ is required in addition to the line parameters $\mathbf{x}_{i}=\left[\epsilon_{i}^{s}, \epsilon_{i}^{\infty}, d_{i}, \rho_{i}, l_{i}\right]$ for each layer $i$. The parameter $l_{i}$ is the length of layer $i$ such that $\sum_{i=0}^{M} l_{i}=L$ where $\mathrm{L}$ is the total length of the M layers.

The analysis again departs from [16] by solving (4) analytically using the physical parameters of the line defined above. Equation (4) is a Riccati differential equation and we have integrated it over a single layer in the $z$ direction from $z_{i+1}$ to $z_{i}$ to yield the recurrence relationship,

$$
r_{i}=\frac{r_{i+1}+A_{i}}{B_{i}-A_{i} r_{i+1}}
$$


where

$$
\begin{gathered}
A_{i}=\frac{P_{i}\left(1-E_{i}\right)}{E_{i}-P_{i}^{2}}, \\
B_{i}=\frac{1-P_{i}^{2} E_{i}}{E_{i}-P_{i}^{2}} \\
P_{i}=-\frac{a_{i}}{b_{i}}+\sqrt{\left(\frac{a_{i}}{b_{i}}\right)^{2}-1}=\frac{-a_{i}+j \omega \sqrt{\epsilon_{i} \mu_{i}}}{b_{i}}
\end{gathered}
$$

and $E_{i}=e^{-j \theta_{i}}$ where $\theta_{i}=2 \omega \sqrt{\epsilon_{i} \mu_{i}} l_{i}$. Given the boundary condition, $r_{M+1}$, that is $Z_{L}$ and $Z_{0}$, and parameters $\mathbf{x}_{i}$, the recurrence relation (11) is used to obtain the solution $r_{0}[12]$.

\section{Development of an Inverse Solver for a Dielectric- Filled Line with a Spatially Varying Geome- try}

In [11], where the line was air-filled, it was assumed that the connector-structure interface could be modelled as a combination of an inductance term and a capacitance term. Unfortunately this is an inadequate model for the connectorstructure interface in a dielectric-filled line. In the former the outer and inner conducting plates are drilled and the signal directly applied in order that the resistance between the connector and plates is kept to a minimum. Attempts have been made to keep the experimental setup as close to a nondestructive testing situation as possible where the drilling of the aircraft aluminium would be out of the question. Nevertheless, regardless of how the signal is applied to the structures it is likely that there will exist some contact resistance between the the connector and the structure.

\subsection{Modelling a Coaxial-Structure Connection: A Four Parameter Approach with Phase Shift}

A schematic diagram of the connection to the dielectric-filled structures is shown in Figure 1. The aforementioned contact resistance is caused by the attachment of the connector to the dielectric-filled structure. The inner conductor of the coaxial cable is attached to the inner plate by a clip and the outer conductor attached to the the outer plate by a securing screw. The build up of aluminium oxide on the outer edges of the structure plates is removed before the structure is attached. Despite these procedures there will still be contact resistances $R_{1}$ 


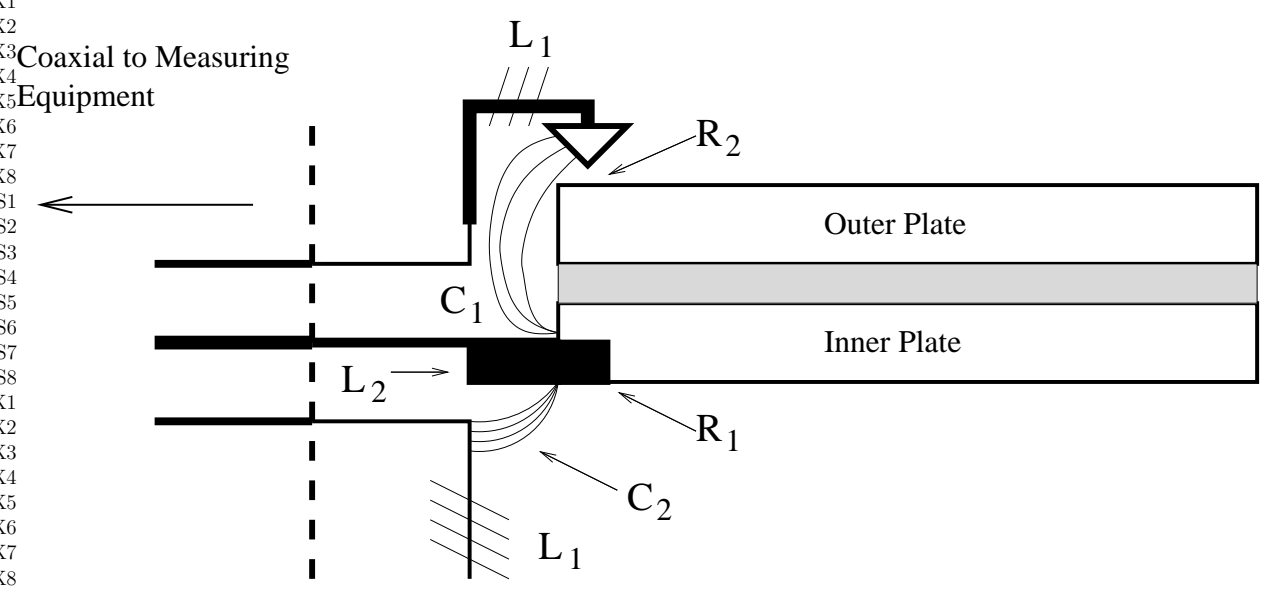

Figure 1: Coaxial-Connector-Plate Construction.

and $R_{2}$ experienced by any signal travelling between the inner plate and the clip and between the outer plate and the securing screw.

The flange will still have an associated inductance $L_{1}$ where the inductive process occurs over the entirety of the flange. In addition to this the inner connecting clip has an inductive contribution $L_{2}$. There will also be an electric field between the flange and the clip attached to the inner plate. An electric field exists between the flange and the clip and therefore a capacitance is present over the entire inductor surface (the flange and the clip). In an attempt to model this simultaneously inductive and capacitive process, two capacitances, $C_{1}$ and $C_{2}$, are used to flank the inductive elements $L_{1}$ and $L_{2}$.

Figure 2 (a) displays the equivalent circuit for the connector; by convention this shows the physical order in which the elements appear. Firstly the line impedance $Z_{L}$ results from the dielectric-filled transmission line. The line is attached to the connector and so two resistances $R_{1}$ and $R_{2}$ are used to represent the contact resistance. The two capacitive elements $C_{1}$ and $C_{2}$ then flank the two inductive elements $L_{1}$ and $L_{2}$ to model the capacitance due to the field between two inductive surfaces.

Figure 2 (b) shows an equivalent circuit to (a) but in an arrangement which allows the impedances to be readily calculated. The two resistors $R_{1}$ and $R_{2}$ are replaced by a single resistor $R$ and similarly $L_{1}$ and $L_{2}$ are replaced by a single inductor $L_{c}$ These new circuit elements are included in the new line parameter $\mathbf{x}_{0}$ and so we write

$$
\mathbf{x}_{0}=\left[R, C_{1}, L_{c}, C_{2}\right]
$$




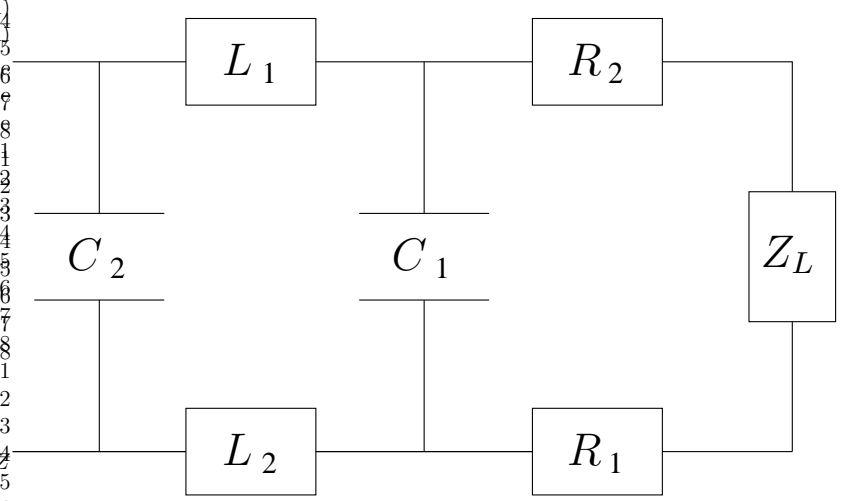

(a) Conventional Circuit: Dual Resistance.

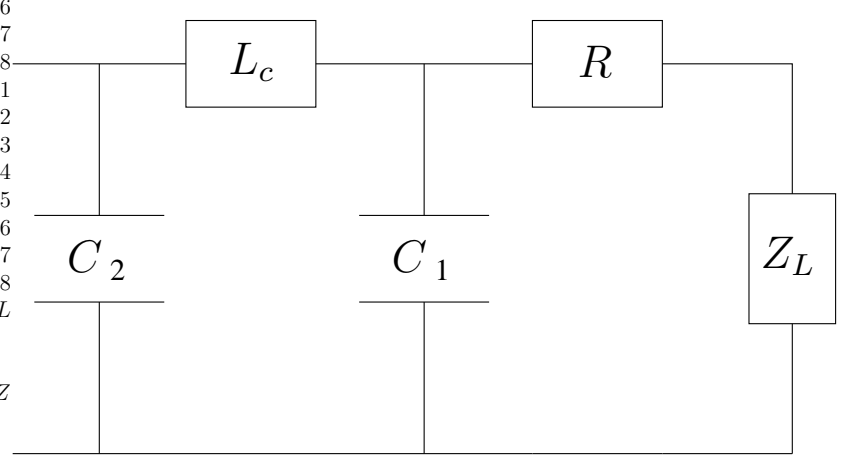

(b) Conventional Circuit: Single Resistance and Inductance.

Figure 2: Equivalent Circuits for Connection to Dielectric-Filled Structures.

The total impedance from this line may now be calculated with the aid of this circuit diagram. The reflection coefficient $r_{0}$ is now given by

$$
r_{0}=\frac{\left[\left(\omega^{2} C_{2} L_{c}-1\right) Z_{0}+i \omega L_{c}\right] Y_{1}+1-i \omega C_{2} Z_{0}}{\left[\left(\omega^{2} C_{2} L_{c}-1\right) Z_{0}-i \omega L_{c}\right] Y_{1}+1+i \omega C_{2} Z_{0}} .
$$

where

$$
Y_{1}=\frac{1}{R+Z_{L}}+i \omega C_{1}
$$

and

$$
Z_{L}=\left(\frac{1+r_{1}}{1-r_{1}}\right) Z_{0}
$$

Once the wave leaves the coaxial-connector interface (and therefore the calibration plane) it must travel some distance $\Delta z$ through the connector before the signal is transferred to the structure. This distance is not accounted for by the 
measuring equipment and thus results in a phase distorted reflection coefficient at the calibration plane. This part of the connector is formed from the coaxial line and therefore may be modelled by a transmission line [11]. So the final reflection coefficient measured at the calibration plane $r_{c a l}$ is given by

$$
r_{c a l}=r_{0} e^{-2 i k \Delta z}
$$

Thus the reflection coefficient seen by the measuring equipment has been shifted in phase by $2 k \Delta z$.

It was shown in [11] that for plates with a finite width the field within the structure shows a slight deviation from that of a uniform field. The potential difference between the plates creates a small fringe field that will be modelled as a single capacitance in parallel with the transmission-line impedance $Z_{L}$. The reflection coefficient resulting from this termination capacitance $C_{t}$ is the boundary condition $r_{b}=r_{M+1}$ in equation (4) and is given by

$$
r_{M+1}=\frac{Z_{\text {term }}-Z_{0}}{Z_{\text {term }}+Z_{0}}
$$

where $Z_{\text {term }}=\frac{1}{i \omega C_{t}}$ is the termination impedance due to capacitance $C_{t}$. The termination capacitance $C_{t}$ gives rise to the reflection coefficient

$$
r_{M+1}=\frac{1-i \omega C_{t} Z_{0}}{1+i \omega C_{t} Z_{0}}
$$

\subsection{Implementation Considerations}

This section will discuss application of the nonlinear least squares approach to the problem of recovering a set of line parameters from an experimentally determined reflection coefficient. The only data available to the inverse solver is that of the reflection coefficient measured at up to 801 separate frequencies in the megahertz to gigahertz range. This data can also be measured from either orientation of the structure and therefore up to 1602 measurements may be made. The objective is to obtain the best least squares solution through solution of

$$
\min _{X \in \mathbb{R}^{n}} f(X)=\min _{X \in \mathbb{R}^{n}} \sum_{k=1}^{N} h_{k}^{2}(X)=\min _{X \in \mathbb{R}^{n}} \mathbf{h}^{\mathbf{T}} \mathbf{h}
$$

for

$$
h_{k}(X)=\frac{1}{\sqrt{N}}\left\{\left|r_{c a l}^{+}\left(X ; \omega_{k}\right)-y^{+}\left(\omega_{k}\right)\right|^{2}+\left|r_{c a l}^{-}\left(X ; \omega_{k}\right)-y^{-}\left(\omega_{k}\right)\right|^{2}\right\} .
$$

The line parameters are contained in

$$
X=\left\{\mathbf{x}_{i}\right\}_{i=1}^{M}
$$




\begin{tabular}{|c|c|c|c|c|}
\hline Electrical Properties & Name & Symbol & Value & Units \\
\hline \hline \multirow{5}{*}{ Connector Properties } & Electrical Length & $\Delta z$ & 14 & $\mathrm{~mm}$ \\
& Connector Resistance & $\mathrm{R}$ & 0.1 & $\Omega$ \\
& Connector Inductance & $L_{c}$ & 1 & $\mathrm{nH}$ \\
& Connector Capacitance 1 & $C_{1}$ & 1 & $\mathrm{pF}$ \\
& Connector Capacitance 2 & $C_{2}$ & 1 & $\mathrm{pF}$ \\
\hline \hline \multirow{5}{*}{ Structure Properties } & Resistivity & $\rho$ & 1 & $\mu \Omega \mathrm{m}$ \\
& Static Permittivity & $\epsilon^{s}$ & 3.5 & - \\
& Infinite Permittivity & $\epsilon^{\infty}$ & 2.5 & - \\
\hline \hline Termination Property & Termination Capacitance & $C_{t}$ & 1 & $\mathrm{pF}$ \\
\hline
\end{tabular}

Table 1: Electrical Property Estimations for Connector, Structure and Termination.

where $\mathbf{x}_{i}$ is the vector

$$
\mathbf{x}_{i}=\left[\epsilon_{i}^{s}, \epsilon_{i}^{\infty}, d_{i}, \rho_{i}\right]^{\mathrm{T}}
$$

containing the line parameters of the $i^{t h}$ layer. There are $M$ layers each with associated vector $\mathbf{x}_{i}$ and $N$ discrete frequencies at which $h_{k}$ must be evaluated. In this case each vector $\mathbf{x}_{i}$ contains the four elements that fulfill a necessary condition for uniqueness [12]. The scaling $\frac{1}{\sqrt{N}}$ in (23) ensures that a comparison of the residuals at the solution $\left(\mathbf{h}^{*}\right)^{\mathrm{T}} \mathbf{h}^{*}$ can be made for problems of a differing number of discrete frequencies $N$. The function (23) makes use of reflection data from both orientations. An analytical expression for the associated Jacobian will also be employed (see Appendix A).

\section{Inversion by Optimisation: Dielectric-Filled Line with Spatially Varying Geometry}

Experimental and simulated data were collected in the form of reflection coefficients for a total of eight structures (labelled X1 to X8) with different plate separations. The structures were manufactured from two pieces of aircraft-grade aluminium bonded by an epoxy resin [8]. Estimates of electrical properties are shown in Tables 1 and 2.

Of the eight structures half were step geometries (X1 to X4) and half double taper geometries (X5 to X8) (See Figure 3). Unfortunately, due to the man- 


\begin{tabular}{|c|c|}
\hline HN Parameter & Value \\
\hline$\tau$ & $5 \mathrm{~ns}$ \\
$\alpha$ & 0.3 \\
$\beta$ & 1 \\
\hline
\end{tabular}

Table 2: Estimated Havriliak-Negami Parameters

\begin{tabular}{|c|c|c|c|c|}
\hline Geometry Type & Structure & Length & Average Width & Average Plate Separation \\
\hline \multirow{3}{*}{ Step } & $\mathrm{X} 1$ & $123.4 \mathrm{~mm}$ & $9.422 \mathrm{~mm}$ & $0.4900 \mathrm{~mm}$ \\
& $\mathrm{X} 2$ & $123.4 \mathrm{~mm}$ & $9.536 \mathrm{~mm}$ & $0.6083 \mathrm{~mm}$ \\
& $\mathrm{X} 3$ & $123.4 \mathrm{~mm}$ & $9.326 \mathrm{~mm}$ & $0.7417 \mathrm{~mm}$ \\
& $\mathrm{X} 4$ & $123.4 \mathrm{~mm}$ & $9.442 \mathrm{~mm}$ & $0.4667 \mathrm{~mm}$ \\
\hline \hline \multirow{3}{*}{ Double Taper } & $\mathrm{X} 5$ & $124.4 \mathrm{~mm}$ & $10.23 \mathrm{~mm}$ & $0.6692 \mathrm{~mm}$ \\
& $\mathrm{X} 6$ & $124.5 \mathrm{~mm}$ & $10.29 \mathrm{~mm}$ & $0.5608 \mathrm{~mm}$ \\
& $\mathrm{X} 7$ & $124.4 \mathrm{~mm}$ & $10.27 \mathrm{~mm}$ & $0.6731 \mathrm{~mm}$ \\
& $\mathrm{X} 8$ & $124.4 \mathrm{~mm}$ & $10.12 \mathrm{~mm}$ & $0.4677 \mathrm{~mm}$ \\
\hline
\end{tabular}

Table 3: Experimentally Determined Structure Dimensions.

\begin{tabular}{|c|c|}
\hline Structure & Plate Separation $(\mathrm{mm}) \pm 10 \%$ \\
\hline X1 & $0.43,0.35,0.38,0.60,0.58,0.60$ \\
X2 & $0.55,0.50,0.50,0.70,0.70,0.70$ \\
X3 & $1.05,0.95,1.00,0.50,0.45,0.50$ \\
X4 & $0.70,0.65,0.70,0.25,0.25,0.25$ \\
\hline \hline X5 & $0.85,0.80,0.70,0.65,0.60,0.55,0.50,0.55,0.60,0.65,0.70,0.75,0.80$ \\
X6 & $0.80,0.75,0.65,0.55,0.48,0.40,0.32,0.35,0.42,0.52,0.60,0.67,0.78$ \\
X7 & $0.85,0.85,0.80,0.65,0.60,0.58,0.54,0.55,0.58,0.60,0.65,0.70,0.80$ \\
X8 & $0.80,0.68,0.55,0.45,0.35,0.27,0.20,0.20,0.30,0.40,0.48,0.60,0.80$ \\
\hline
\end{tabular}

Table 4: Experimentally Determined Plate Separations. 


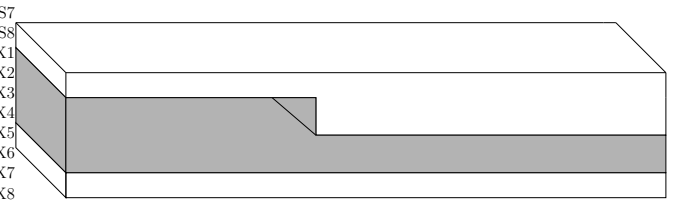

(a) Step Geometry.

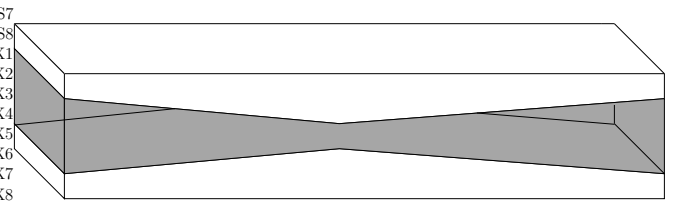

(b) Double Taper Geometry.

Figure 3: Structure Geometries.

ufacturing process only the length could be prescribed accurately. The width of each structure showed variations of up to $0.1 \mathrm{~mm}$ along its length. For this reason the width of each structure was measured (in millimeters to an accuracy of 2 decimal places) at intervals along its length and an average value taken. Further complications arose in the measurement of the plate separation. A microscope was used to measure the separation between the plates (in millimeters to an accuracy of 2 decimal places) along the length of the structure. Despite this effort, the accuracy could not be guaranteed due to surface variations on the aluminium (see Tables 3 and 4 ).

The values displayed in Tables 1-4 (not including the average plate separation) were used to generate simulated reflection coefficient data by employing the layered-model (11). Therefore the structures X1 to X8 could be regarded as having simulated counterparts S1 to S8 each with associated reflection coefficients. These fictitious structures were generated using spatially varying plate separations along the structure length but with the width chosen simply to be the average width (See Tables 3 and 4). The step structures S1 to S4 were each generated using 6 layers while the double-tapered structures S5 to S8 were generated using 13 layers.

\subsection{Parameter Recovery From Simulated Data: Dielectric- Filled Transmission Line}

Parameter recovery from simulated data will be reported first. The optimisation problem was solved for iteration stopping tolerances

$$
\text { tol }=\left|f^{k}-f^{k+1}\right|<\left\{\begin{array}{l}
10^{-10} \\
10^{-16}
\end{array}\right.
$$

using data collected over the full available frequency range $7.5 \mathrm{MHz}$ to $6 \mathrm{GHz}$ (801 equally spaced data points). As previously discussed the recovery from experimental data was often poor when high frequency measurements were involved. For this reason parameter recovery is also considered in the low to mid frequency range $7.5 \mathrm{MHz}$ to $3 \mathrm{GHz}$ (401 equally spaced data points).

The solver was set to recover the 9 electrical parameters specified in Table 1 as well as the plate separation of each layer. The number of layers used by the 


\begin{tabular}{|c|c|c|c|c|}
\hline Property Type & Parameter & Lower Bound & Upper Bound & Units \\
\hline \hline \multirow{4}{*}{ Connector Properties } & $\Delta Z$ & 13 & 17 & $\mathrm{~mm}$ \\
& $R$ & $10^{-4}$ & 100 & $\Omega$ \\
& $L_{c}$ & 0.01 & 1000 & $\mathrm{nH}$ \\
& $C_{1}$ & 0.01 & 1000 & $\mathrm{pF}$ \\
& $C_{2}$ & 0.01 & 1000 & $\mathrm{pF}$ \\
\hline \hline & $\epsilon^{\infty}$ & 2 & 3.5 & - \\
Structure Properties & $\epsilon^{s}$ & 3 & 4.5 & - \\
& $\rho$ & $10^{-4}$ & 100 & $\mu \Omega \mathrm{m}$ \\
\hline \hline Termination Property & $C_{t}$ & 0.01 & 100 & $\mathrm{pF}$ \\
\hline \hline Geometry Property & $d_{i}$ & 0.01 & 10 & $\mathrm{~mm}$ \\
\hline
\end{tabular}

Table 5: Lower and Upper Bounds

inverse solver was set in the first instance to that used to generate the simulated data (that is 6 for structures S1 to S4 and 13 for structures S5 to S8). In a real NDT situation the number of layers will not be known and indeed the structure may not even be of a layered type. Such a case will be illustrated by recovering plate separations of the double taper structures X5 to X8 where there is no obvious layer arrangement (see Figure 3). In order to overcome this unknown factor the inverse solver was also set to recover plate separations using 10, 15 and 20 layers. This can be thought of as increasing the resolution of the reconstruction.

The initial conditions for the electrical properties were chosen randomly from a uniform distribution which lay within $5 \%$ of those detailed in Table 1. For the plate separation initial condition, every layer was set to the average plate separation (see Table 3). This was deemed a reasonable approximation to what would be known a priori in an NDT situation. During the optimisation process lower and upper constraints were set on the reconstruction parameters (see Table 5). All other parameters were assumed to be known and therefore set to their exact value (see Tables 2 and 3 ).

\subsubsection{Results: Geometry Recovery}

Firstly the solver was supplied with exact layer information, that is to say the inverse solver was supplied with the exact location and length of the layers used 


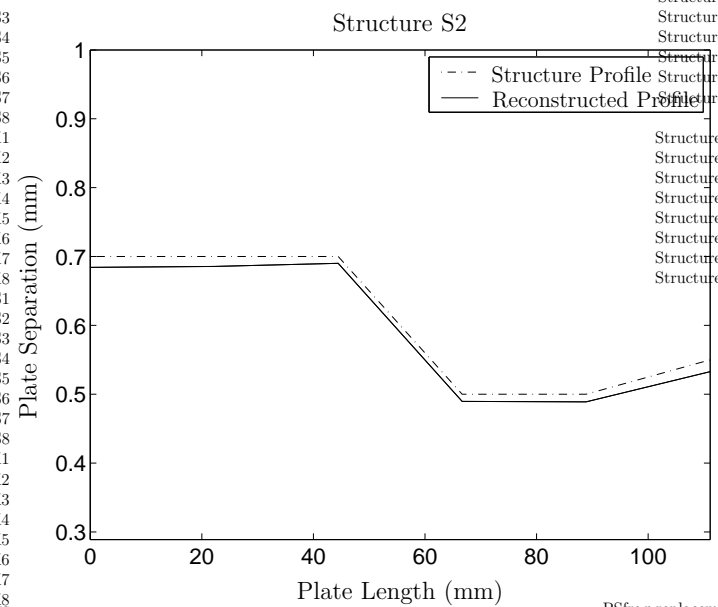

(a) Structure S2 with Stopping

Tolerance: $t o l=10^{-10}$.

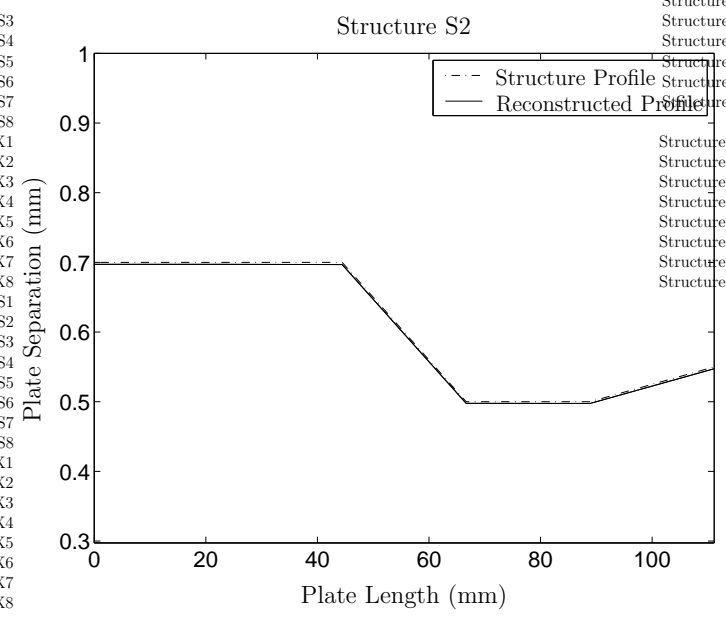

(c) Structure S2 with Stopping

Tolerance: $t o l=10^{-16}$.

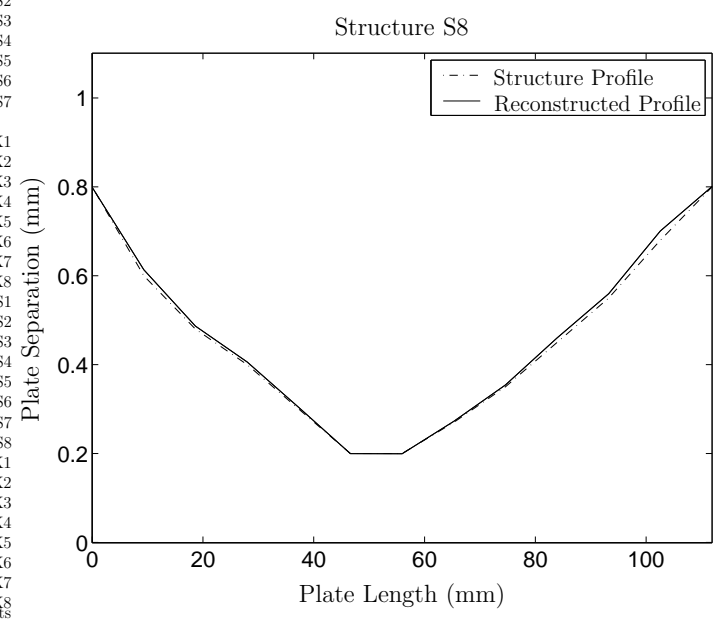

(b) Structure S8 with Stopping Tolerance: $t o l=10^{-10}$.

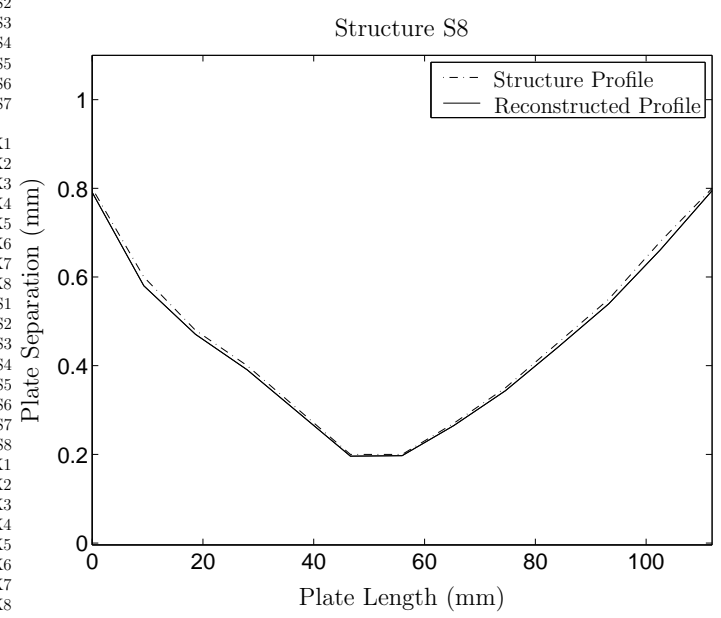

(d) Structure S8 with Stopping Tolerance: tol $=10^{-16}$.

Figure 4: Reconstructions for Varying Tolerances.

in the generation of the simulated data; that is structure S2 was reconstructed using 6 layers and structure S8 using 13 layers. The problem was solved for both tolerances in equation (26) (see Figure 4). Plots (a) to (d) all show acceptable reconstructions of the plate separation profile. In particular Plots (c) and (d) show that using tol $=10^{-16}$ is sufficient to obtain an almost exact reconstruction of the step structure S2 as well as an extremely good reconstruction for the tapered structure S8. For this reason all subsequent iterations were run to a tolerance of $t o l=10^{-16}$.

In general the data supplied to the solver will not be from layered media but 


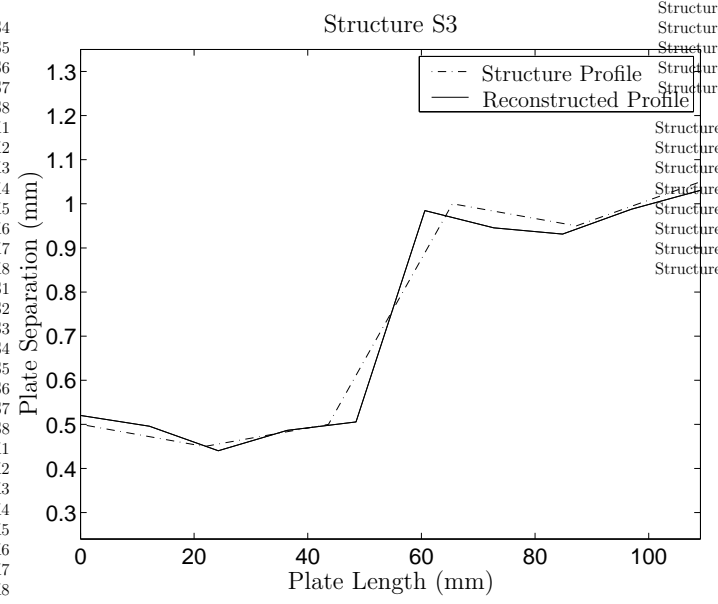

(a) Structure S3: 10 Layers.

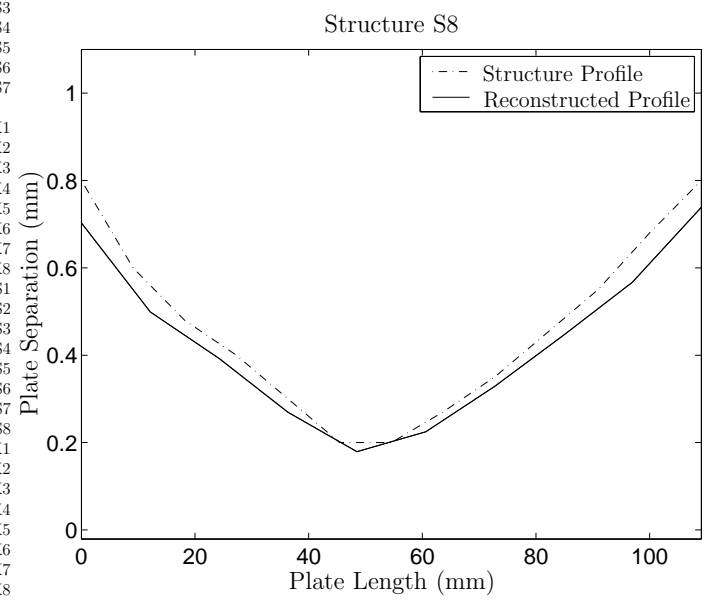

(b) Structure S8: 10 Layers.

Figure 5: 10 Layer Reconstructions for Structures S3 and S8.

from media with a continuously changing geometry. The layered-model attempts to reconstruct a discrete approximation to this continuous geometry case. The number of layers specified may be interpreted as the degree of spatial resolution. Reconstructions were first made with a resolution of 10 layers. This model is really an overestimation of the number of layers required for the structures S1 to S4 and an underestimation for the structures S5 to S8 (see Figure 5). Plots (a) and (b) display successful reconstructions. Quantitatively the reconstructions compare favourably with the original profile and perhaps more importantly the reconstructions also agree qualitatively with the original profile.

On increasing the resolution to 15 layers it can be seen that reconstructions begin to oscillate about the true solution for step profile S3 (see Figure 6). The reconstruction in Plot (a) still displays reasonable qualitative behaviour with the step correctly positioned. Oscillations are not observed in Plot (b) where the smoother profile of structure S8 is successfully reconstructed without any visible oscillations. Upon increasing to 20 layers Plot (c) shows that the oscillatory behaviour in the reconstruction of structure S3 increases, however Plot (d) shows that the reconstruction of the tapered profile of structure S8 still compares well with the original.

It was thought that, due to the static approximations made in Section 2, the model may be less accurate at utilising high frequency data and for this reason reconstructions were attempted using the low to mid range frequency data (401 equally spaced data points from $7.5 \mathrm{MHz}$ to $3 \mathrm{GHz}$ ) (see Figure 7).

It is clear from Plots (a) to (d) that geometry reconstruction for the low to mid range frequency data is less successful than that for the full range of frequency data. Plots (a) and (c) show good quantitative behaviour for both 10 


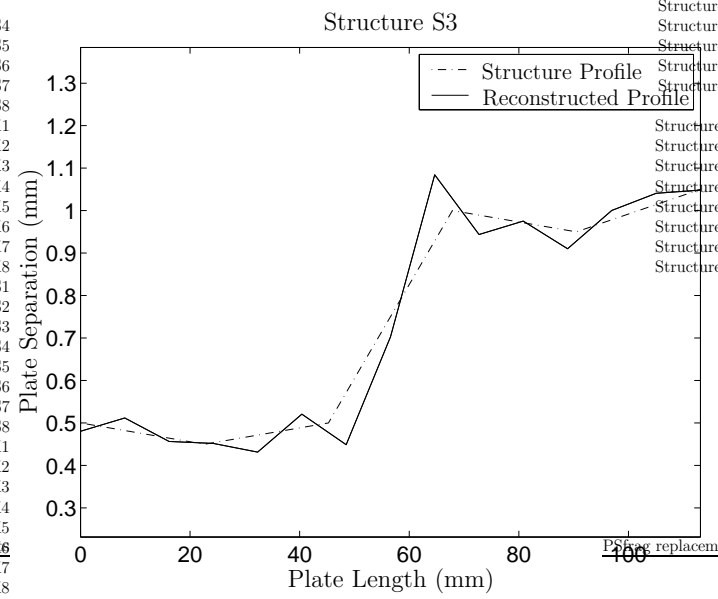

(a) Structure S3: 15 Layers.

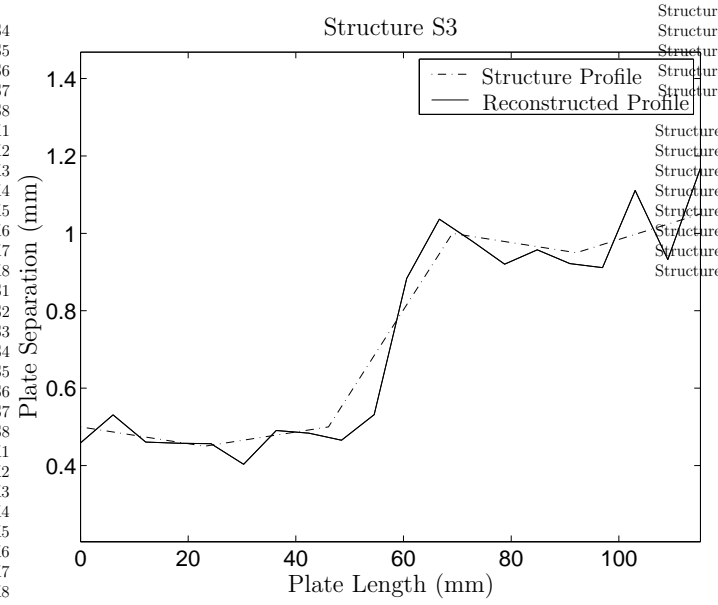

(c) Structure S3: 20 Layers.

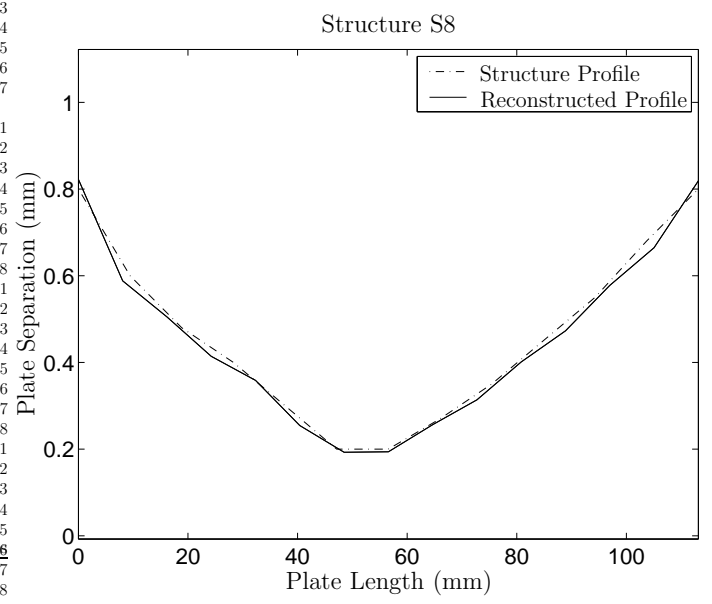

(b) Structure S8: 15 Layers.

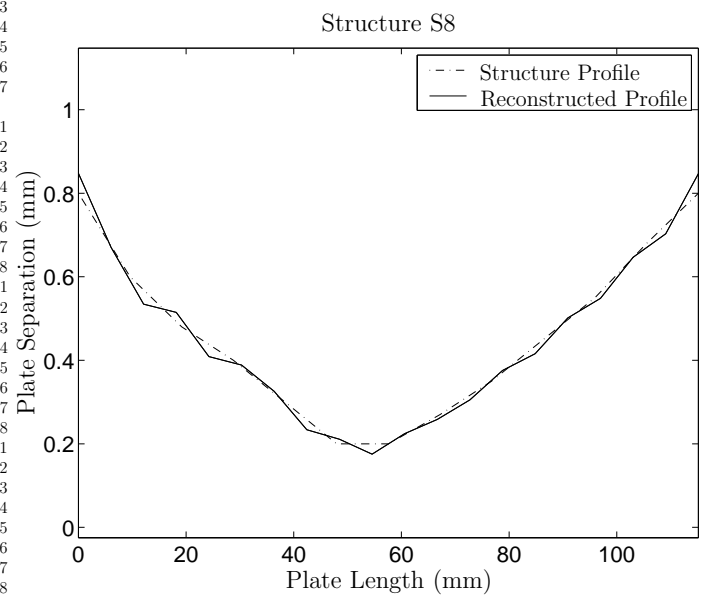

(d) Structure S8: 20 Layers.

Figure 6: 15 and 20 Layer Reconstructions for Structures S3 and S8.

and 20 layer reconstructions of stepped structure S3. However, oscillations have now appeared in the 10 layer resolution where previously smooth reconstructions were observed. Plot (b) displays an acceptable 10 layer reconstruction for the tapered structure S8 whereas Plot (d) shows a poor 20 layer reconstruction. Reconstructions for 15 layers display similar qualities to that of 10 layers for both structures S3 and S8.

It would therefore appear that the full range of frequency data is required to retrieve the geometrical information to an acceptable accuracy. However, it may be that a satisfactory recovery of electrical properties can be achieved from low to mid range data. 


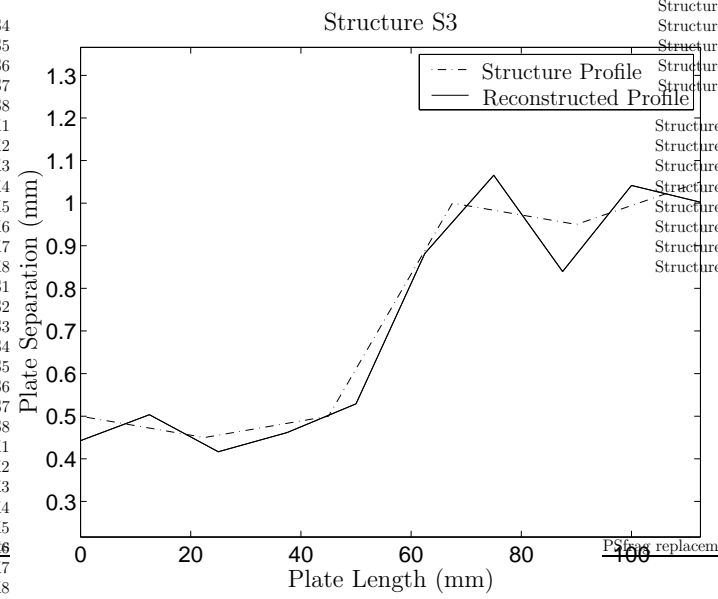

(a) Structure S3: 10 Layers.

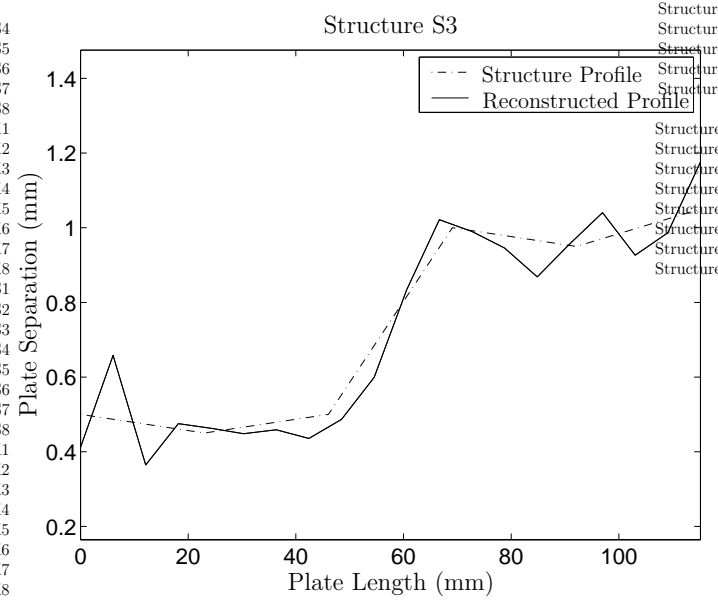

(c) Structure S3: 20 Layers.

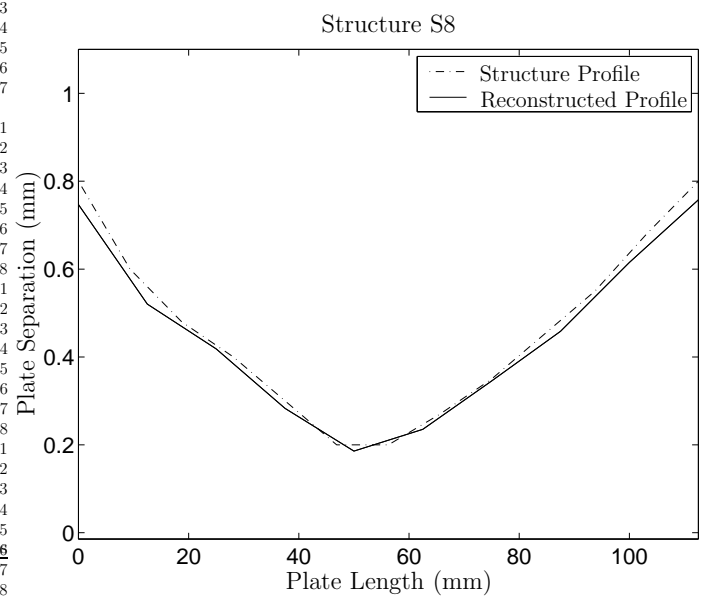

(b) Structure S8: 10 Layers.

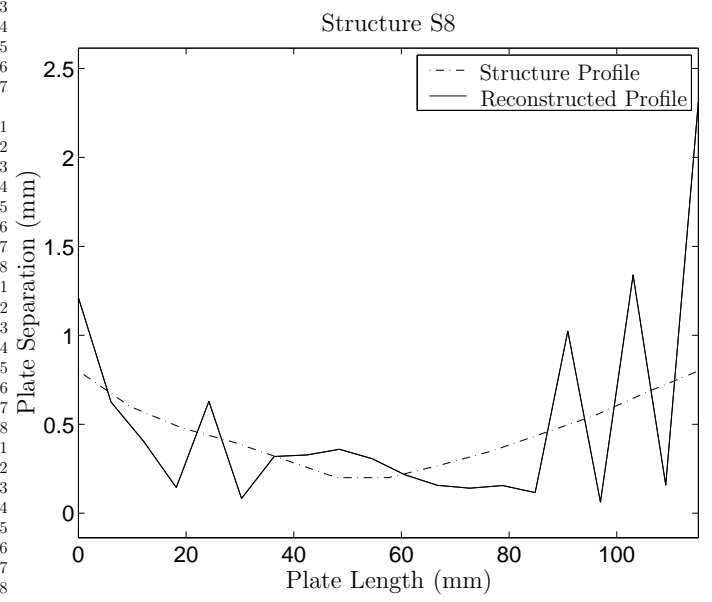

(d) Structure S8: 20 Layers.

Figure 7: 10 and 20 Layer Reconstructions for Structures S3 and S8: Low to Mid Frequency.

\subsubsection{Results: Electric Property Recovery}

Electrical properties were also reconstructed from simulated data (generated using the values in Table 1). The most important of these electrical properties are the resistivity and permittivity as the value of these properties can give an indication of the bond strength between the aluminium plates.

Reconstructions were obtained using a model supplied with the exact layer location and length used to generate the simulated data (see Figure 8). Plots (a) and (b) show an acceptable reconstruction for the permittivity with stepped structures outperforming tapered structures. Plot (c) however displays a reconstruction of the resistivity far removed from the correct value of $1 \mu \Omega \mathrm{m}$. Recon- 


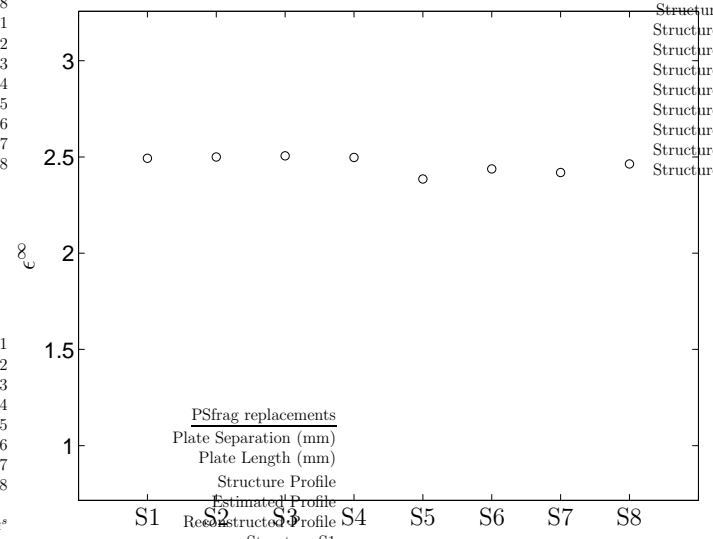

(a) Infinite Permittivity Reconstruction.

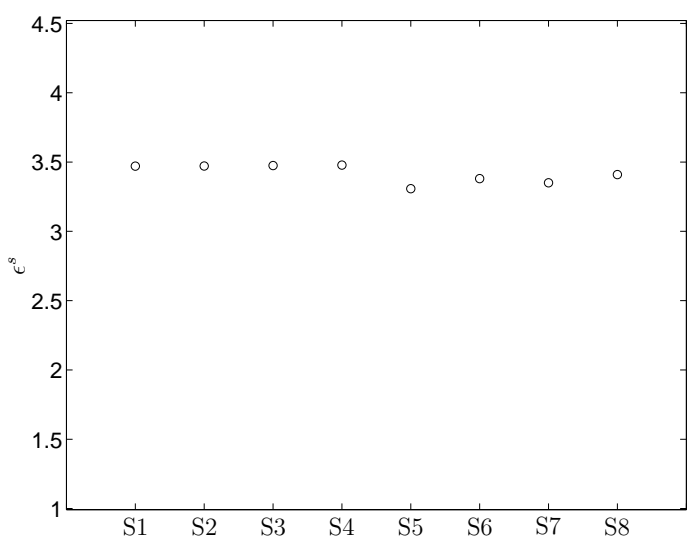

(b) Static Permittivity Reconstruction.

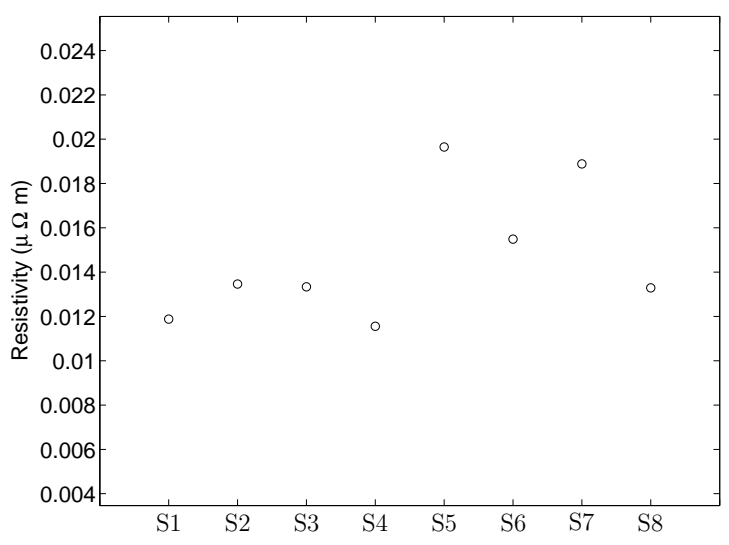

(c) Resistivity Reconstruction.

Figure 8: Reconstructed Structural Electrical Properties of S1 to S8: Exact Layers.

structions of the connector inductance, capacitance and resistance (not shown here for brevity) were also obtained and found to display similar inaccuracies and inconsistences to that of the resistivity reconstruction [12].

Reconstructions of electrical properties were also made for simulated data where the number of layers was chosen to be 10, 15 and 20 (see Figure 9). Plots (a) and (b) again show good reconstructions for both infinite and static permittivities. Plot (c), however, still displays inaccurate and inconsistent reconstructions for the resistivity.

Figure 10)(a) displays an acceptable reconstruction of the electrical length for all structures and resolutions. Plot (b) however displays an inaccurate reconstruction of the termination capacitance: it is clearly overestimated for all structures, and indeed there are also inconsistencies between reconstructions. 


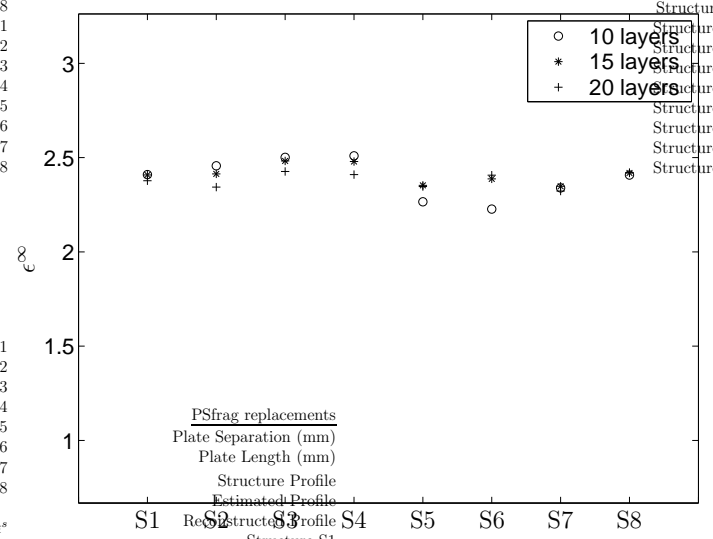

(a) Infinite Permittivity Reconstruction.

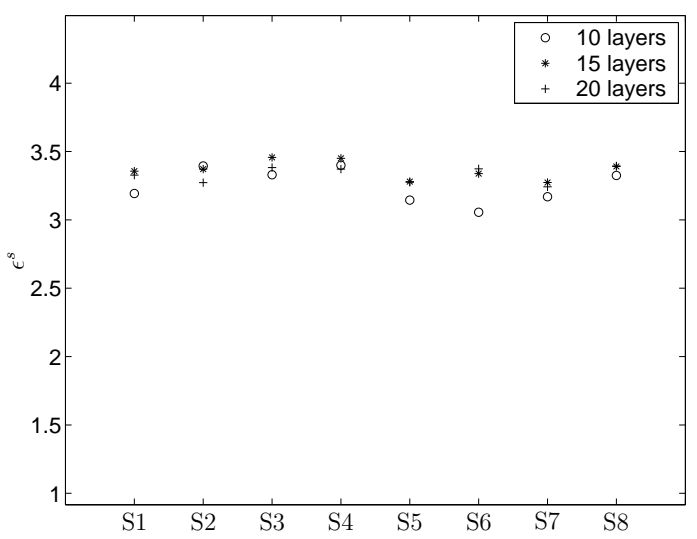

(b) Static Permittivity Reconstruction.

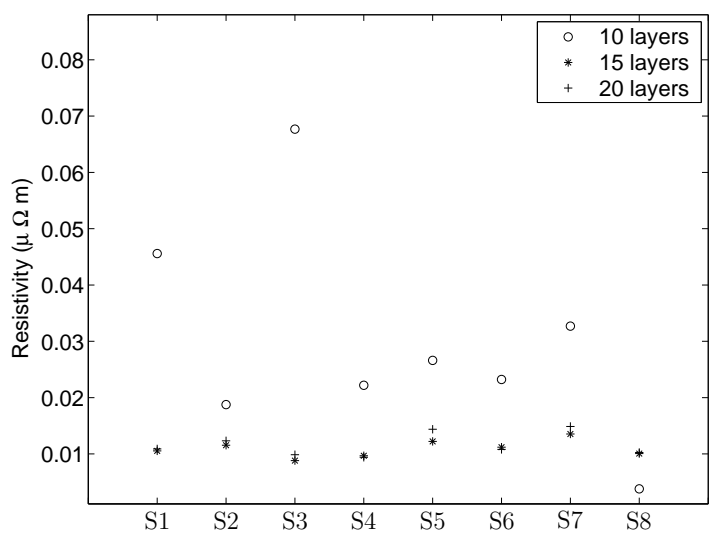

(c) Resistivity Reconstruction.

Figure 9: Reconstructed Structural Electrical Properties of S1 to S8: 10, 15 and 20 Layers.

Similar problems were again observed in reconstructions for the connector parameters, none of which were successfully resolved. It may be that these inaccuracies are linked to the poor resistivity reconstructions.

\subsection{Parameter Recovery From Experimentally Recovered Data: Dielectric-Filled Transmission Line}

The ultimate aim of the study has been to construct a model which may be employed to recover electrical and in particular geometrical parameters of an aluminium-epoxy-aluminium structure using experimentally recovered reflection coefficients. In this section reconstructions of these electrical and geometrical parameters will be presented and discussed. 


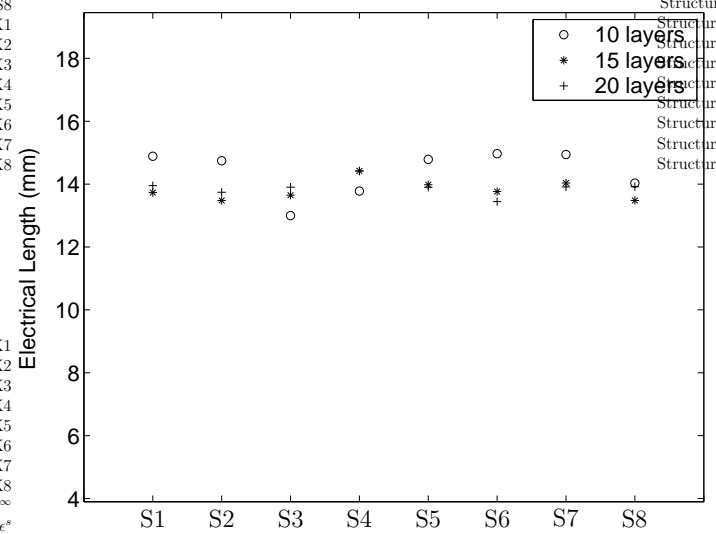

(a) Electrical Length Reconstruction.

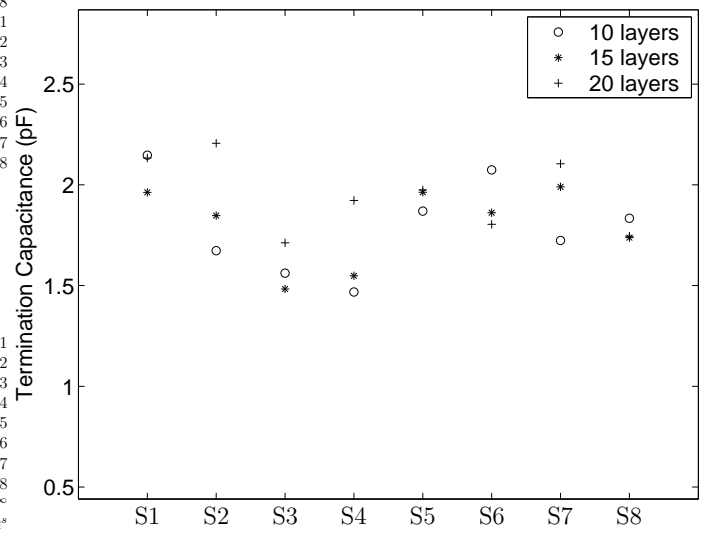

(b) Termination Capacitance Reconstruction.

Figure 10: Reconstructed Electrical Length and Termination Capacitance of S1 to S8: 10, 15 and 20 Layers.

Reflection coefficients from the full frequency range $7.5 \mathrm{MHz}$ to $6 \mathrm{GHz}(801$ equally spaced data points) and from the low to mid frequency range $7.5 \mathrm{MHz}$ to $3 \mathrm{GHz}$ (401 equally spaced data points) were employed. The 9 electrical parameters recovered using simulated data were again recovered using the experimental data (see Table 1). In addition to this plate separations were recovered for resolutions of 10, 15 and 20 layers. The values of all other parameters were set to the estimates given in Table 2 and 3. It should be emphasised that the values in Table 2 are only rough estimates taken from experimental evidence independent of reflection coefficient recovery. It should also be noted that although the value for the structure width is taken from experimental measurements it actually represents an average width; again as a consequence of the manufacturing limitations. The model assumes that these parameter estimations are exact and thus errors are introduced before data noise and modelling assumptions are considered.

\subsubsection{Results: Geometry Recovery}

In this section plate separation reconstructions will be presented. The reconstructed plate separations will be compared to the corresponding physical measurements. Although uncertainties arise in the quantitative measurement of the plate separations this is not the case qualitatively and it may be confidently stated that structures X1 to X4 are of step type whereas structures X5 to X8 are of double taper type (see Figure 3 ).

Reconstructions at the 10 layer resolution are extremely close to those estimated by the experimental measurement (see Figure 11). Importantly Plots (a) 


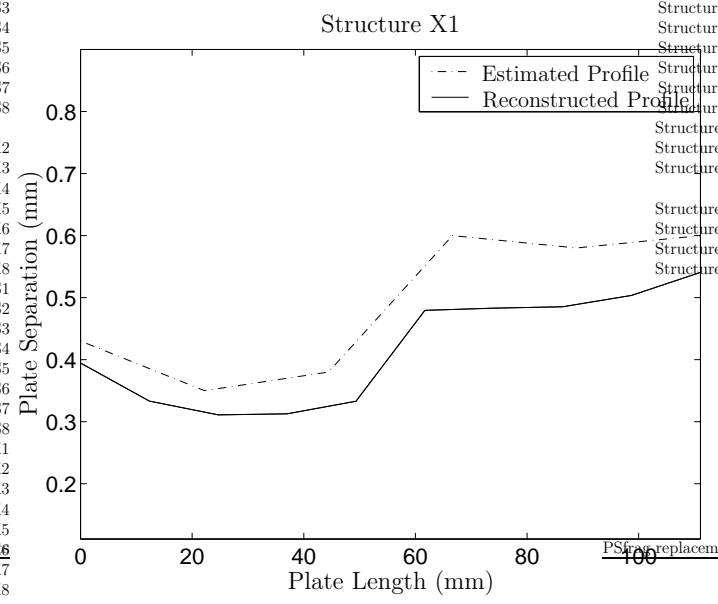

(a) Structure X1: 10 Layers.

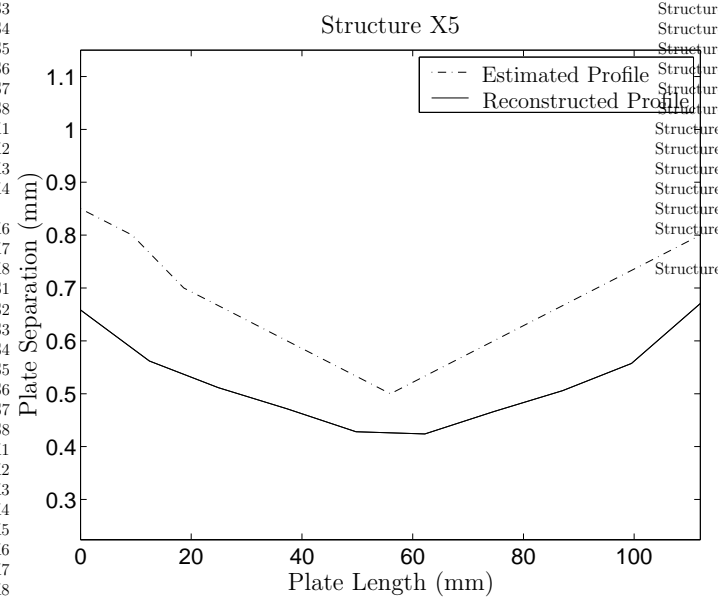

(c) Structure X5: 10 Layers.

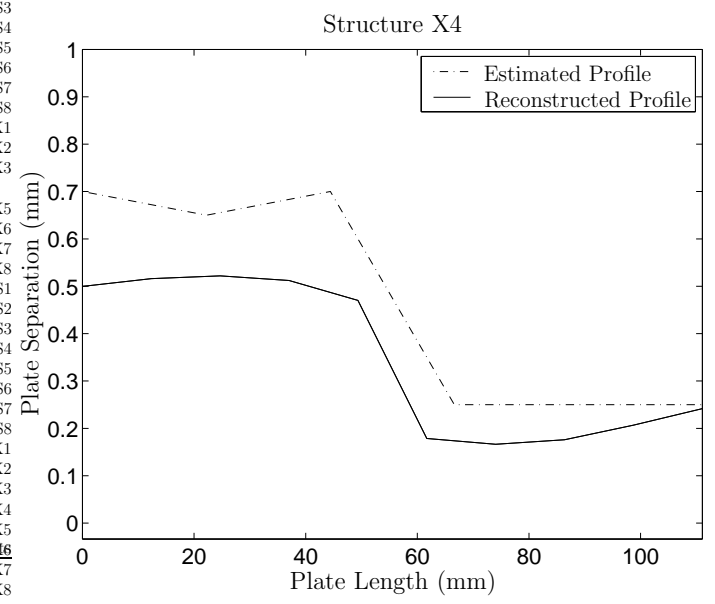

(b) Structure X4: 10 Layers.

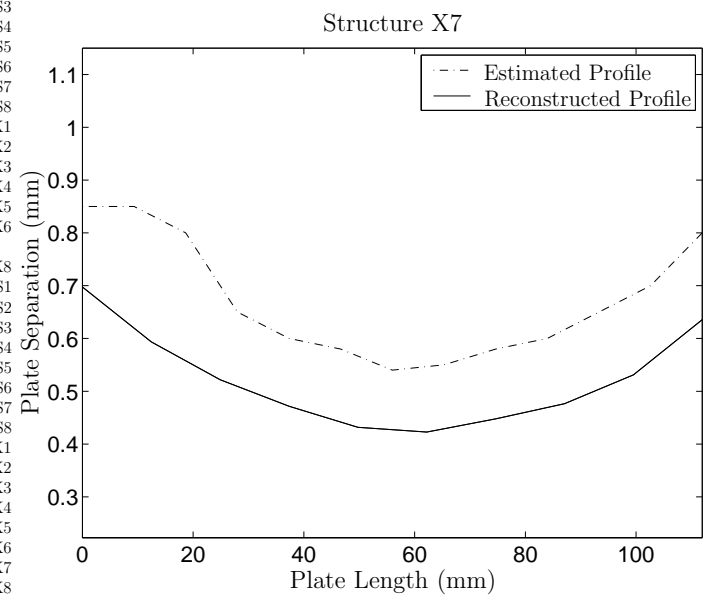

(d) Structure X7: 10 Layers.

Figure 11: 10 Layer Reconstructions (note scale).

and (b) agree qualitatively with the estimated profile (note that the step occurs in the correct position). Plots (c) and (d) also agree qualitatively with the estimated profile with the double taper geometry clearly visible. However quantitatively the reconstructions consistently underestimate the plate separation by approximately $0.2 \mathrm{~mm}$. Nevertheless the experimentally obtained profiles are themselves only estimates and the consistency with which they are underestimated suggests that adjustment of another parameter may compensate for this.

At a resolution of 15 layers the reconstructions are not quite as smooth as they were for 10 layers (see Figure 12). However Plots (a) to (d) still display good agreement with the experimental estimates. The consistent underestimation of 


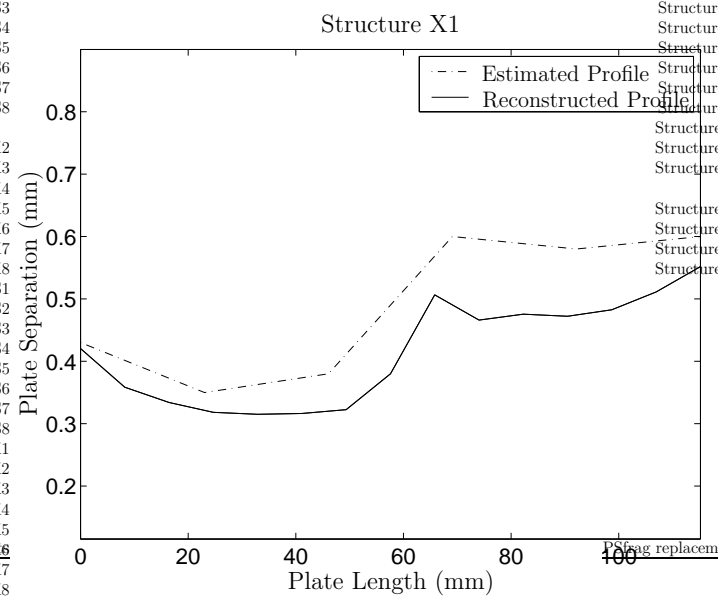

(a) Structure X1: 15 Layers.

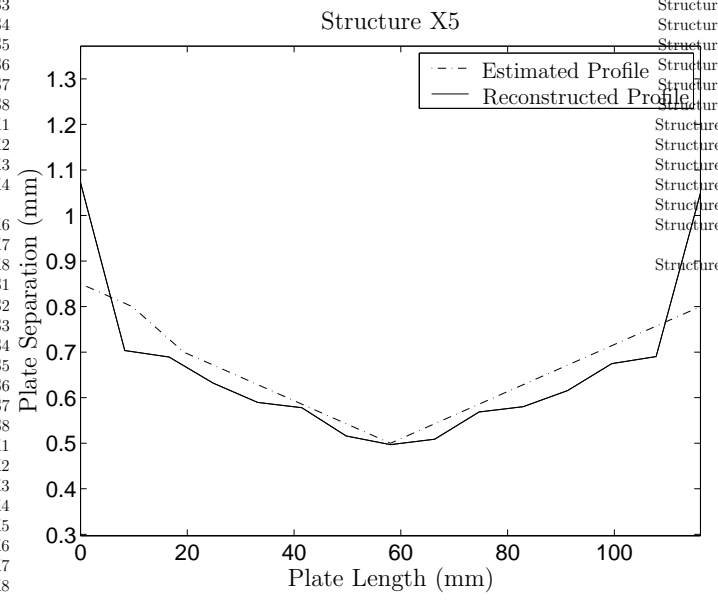

(c) Structure X5: 15 Layers.

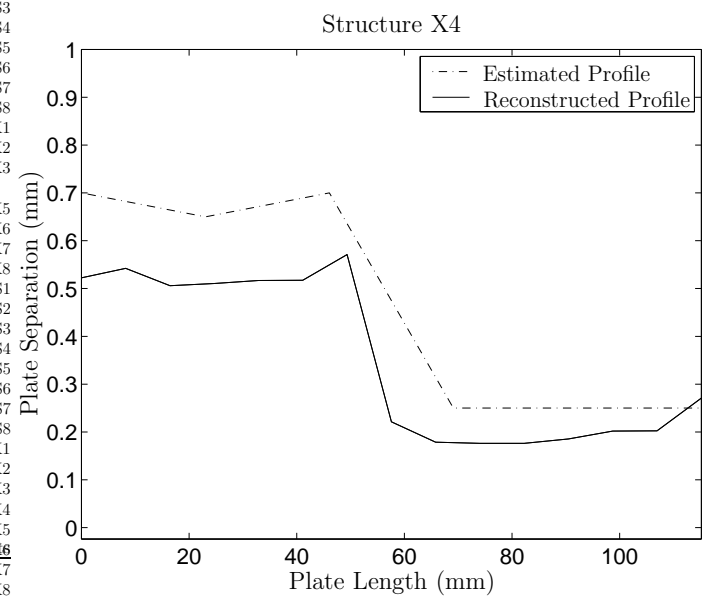

(b) Structure X4: 15 Layers.

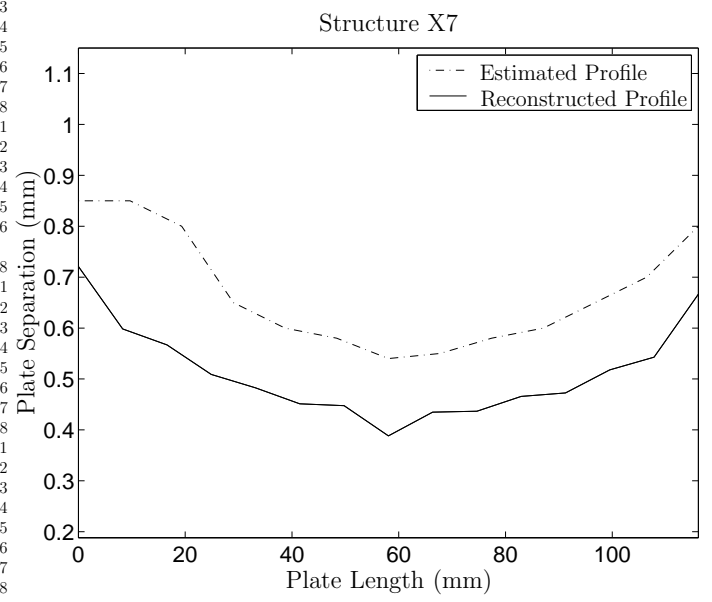

(d) Structure X7: 15 Layers.

Figure 12: 15 Layer Reconstructions.

the plate separation is again prevalent in all but Plot (c) where only the end points have been overestimated.

For a resolution of 20 layers and above, oscillations start to dominate the reconstructions (see Figure 13). Plots (a) to (d) all display oscillations in both step and tapered type structures. The reconstruction oscillates about what appears to be (at least qualitatively) something close to the estimated plate separation. The reconstructions obtained using the simulated data suggestedthat data noise or modelling assumptions are not the principal causes of such oscillations (see Figure 6). It may be that increasing the number of variables in the optimisation routine has affected the reconstructions.

A possible cause of the consistent underestimation of the plate separation is 


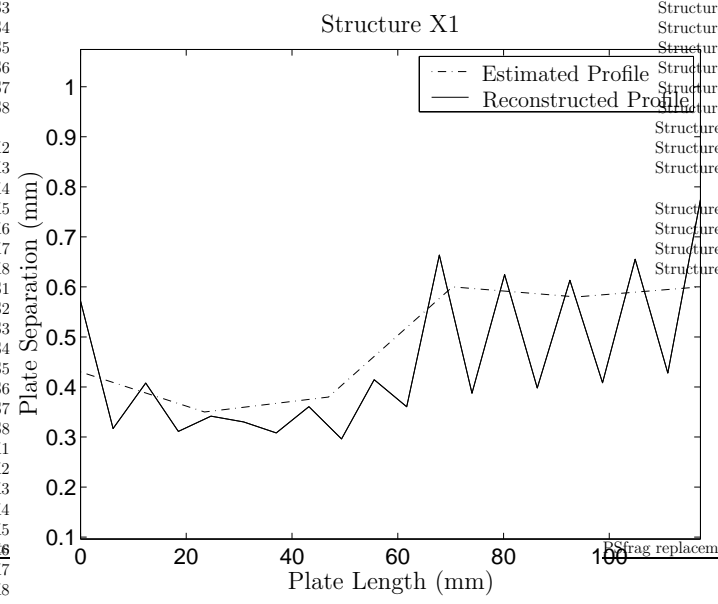

(a) Structure X1: 20 Layers.

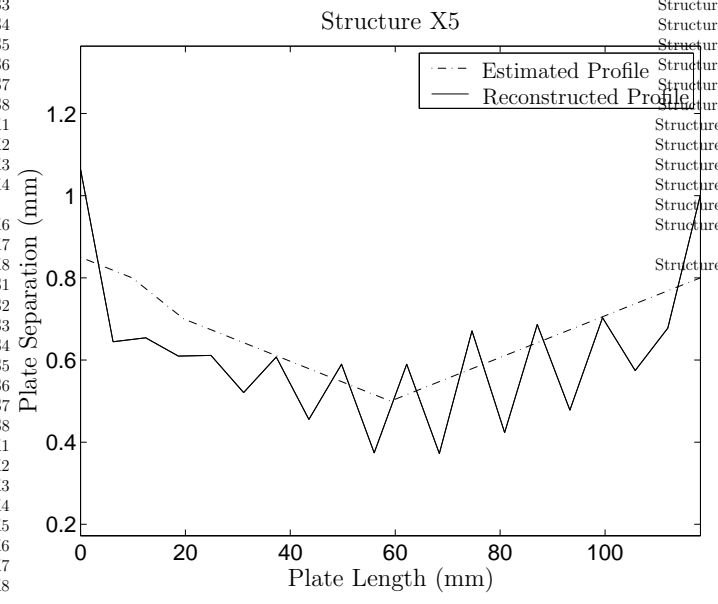

(c) Structure X5: 20 Layers.

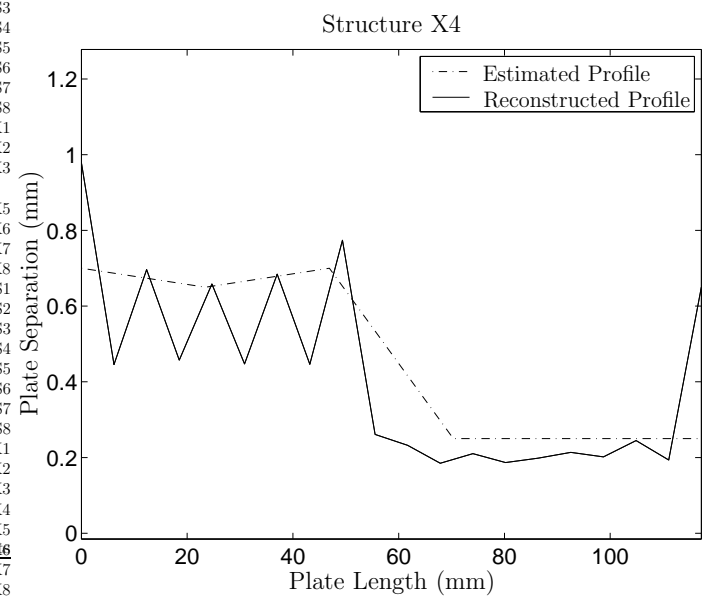

(b) Structure X4: 20 Layers.

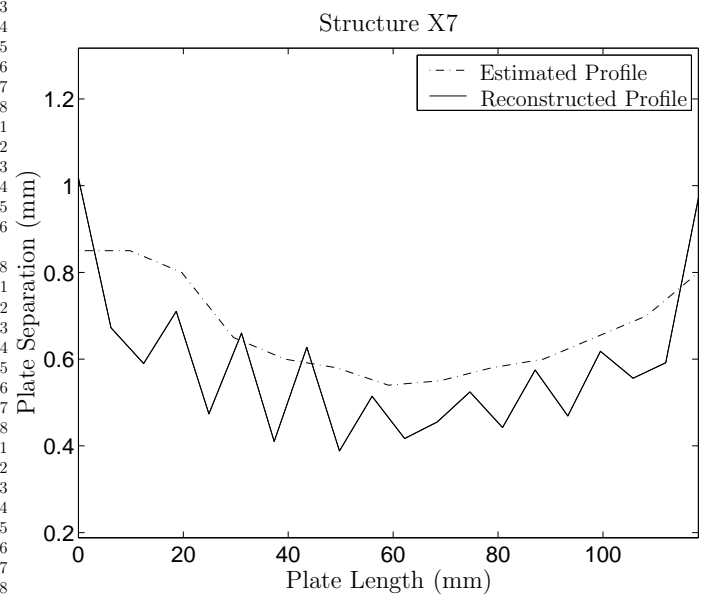

(d) Structure X7: 20 Layers.

Figure 13: 20 Layer Reconstructions.

that of a fringe field. In [11] a fringe field was introduced into the model for the air-filled line. It was found that the reconstruction of the physical width yielded a value close to that of the effective width predicted by finite element calculations of the two dimensional Maxwell equations. In the case of the dielectric-filled structures it was thought that such fringe fields would be negligible and would have little effect on the recovered reflection coefficient.

Increasing the average width of each structure supplied to the inverse solver by $25 \%$ increases the plate separation (see Figure 14). Plots (a) to (d) all show reconstructions which quantitatively are within $0.1 \mathrm{~mm}$ of the experimental estimates. Note that these reconstructions are more or less identical to that of Figure 11 but with the plate separation increased by a fixed amount. Reconstruc- 


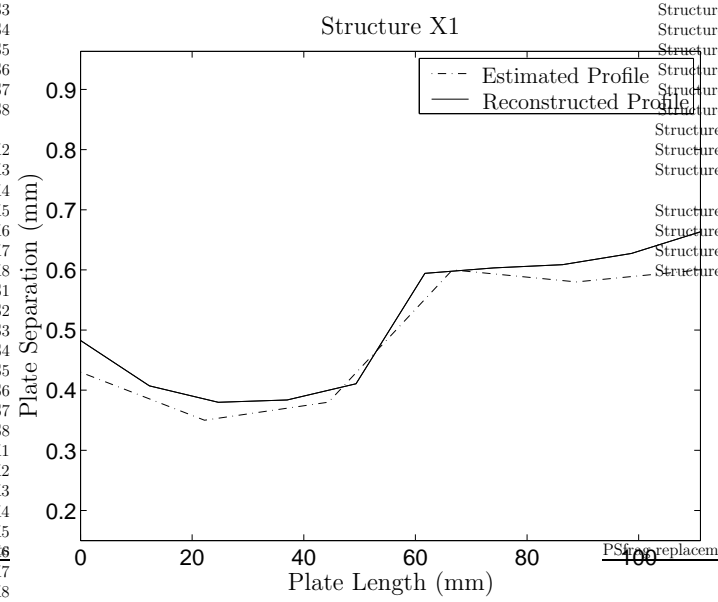

(a) Structure X1: 10 Layers.

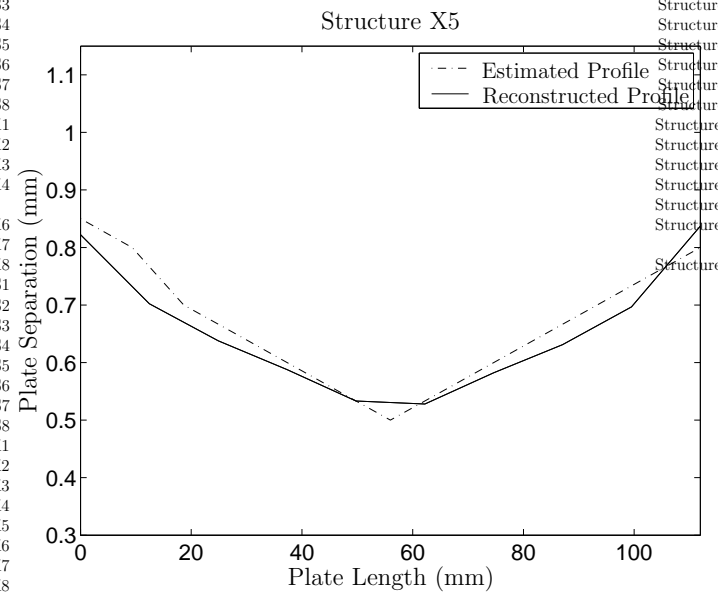

(c) Structure X5: 10 Layers.

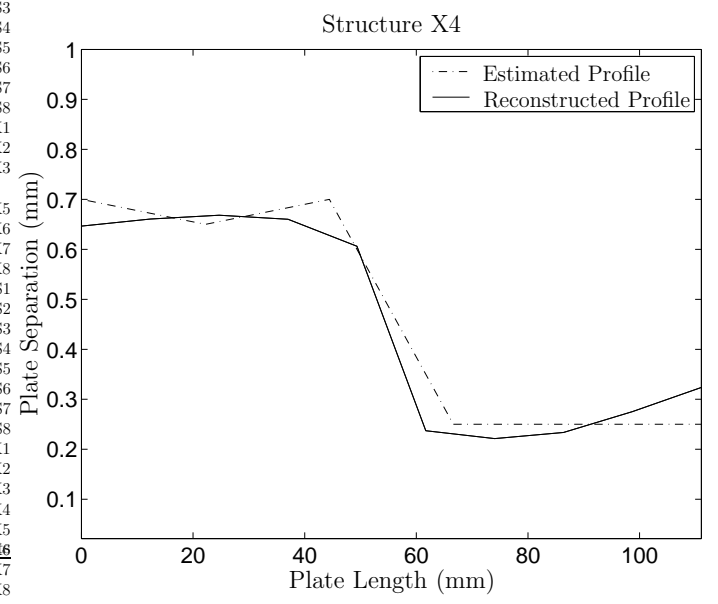

(b) Structure X4: 10 Layers.

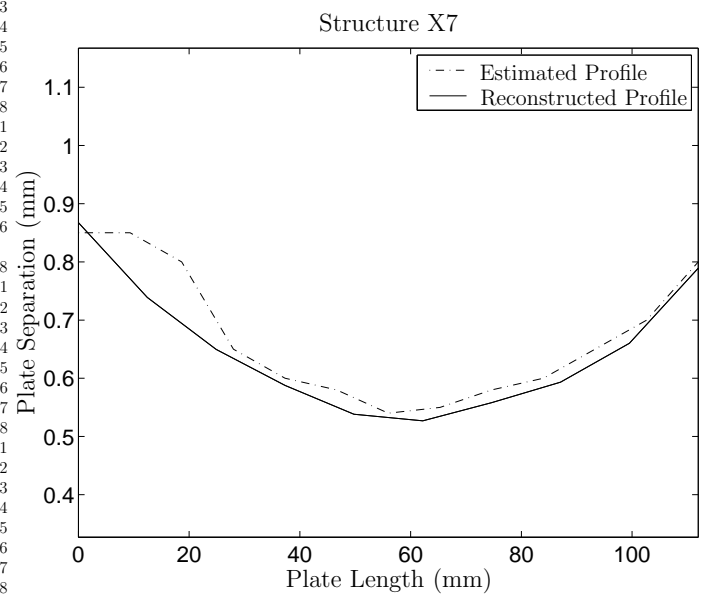

(d) Structure X7: 10 Layers.

Figure 14: 10 Layer Reconstructions For an Effective Width $W \rightarrow 1.25 \times W$.

tions at higher resolutions again show similar results with a consistent increase in the average plate separation.

Unfortunately a similar finite element analysis to that of [11] does not suggest that the effective width is $25 \%$ greater than the true width. Note also that the width cannot be included as an unknown line parameter as this violates the uniqueness conditions discussed in [11]. It is unlikely that the experimental measurements of the width could be so inaccurate and so a more plausible explanation is that the underestimation of the plate separation is compensating for some error in an electrical parameter. 


\subsubsection{Results: Electric Property Recovery}

In this section reconstructions of the electrical properties will be presented. Unlike the geometry recovery there are no precise values for these electrical properties and so reconstructions may only be judged against their estimates (see Table 1). Physical considerations provide a feasible region for the parameters (see Table 5).

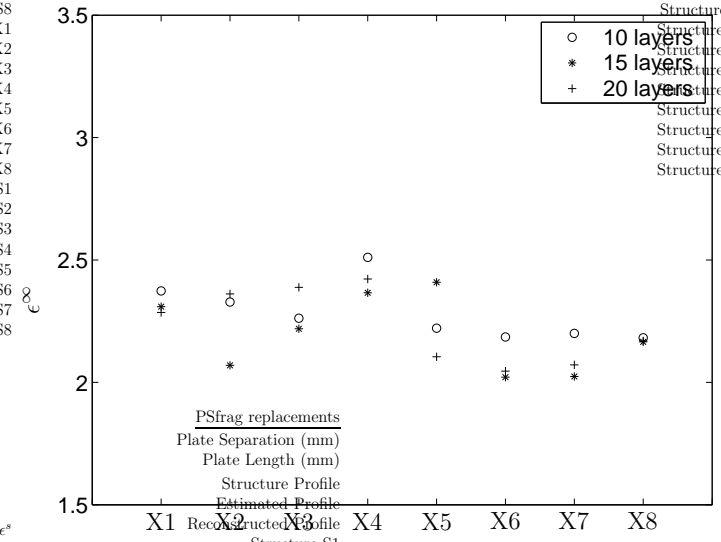

(a) Infinite Permittivity Reconstruction.

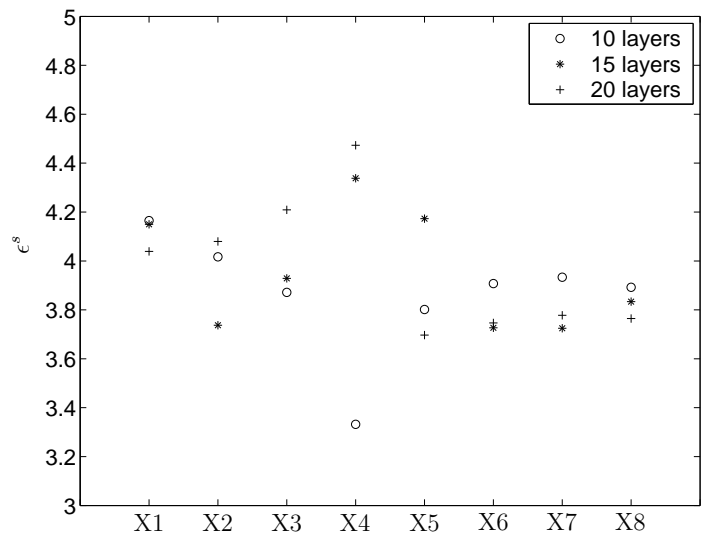

(b) Static Permittivity Reconstruction.

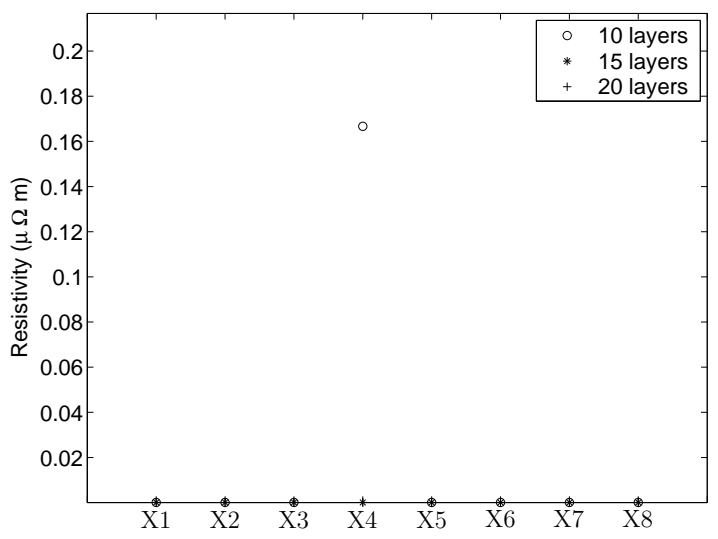

(c) Resistivity Reconstruction.

Figure 15: Reconstructed Structural Electrical Properties of X1 to X8: 10, 15 and 20 Layers.

In Figure 15, Plots (a) and (b) display reconstructions of both infinite and static permittivities for structures $\mathrm{X} 1$ to $\mathrm{X} 8$ which are relatively consistent with those recovered from simulated data (see Figure 10). The range of permittivities displayed are physically feasible although the static permittivity reconstructions are slightly high and there are unexpected variations between structures. There 
are other points for concern, in particular the number of layers used to reconstruct the geometry appears to affect the electrical reconstructions. This is highlighted in Plot (b) where the static permittivity reconstructions of structure X4 vary according to the number of layers used for geometry reconstruction. This suggests problems in the uniqueness of the reconstruction since different initial conditions are iterating to different solutions. The reconstructions of the resistivity displayed in Plot (c) show a divergence, in all but one reconstruction, to the lower constraint boundary. It is highly unlikely that the resistivity of the aluminium should be so low and again it clear from structure X4 that reconstructions are also inconsistent. Indeed it is possible that the apparent underestimation of the 10 layer static permittivity reconstruction for structure $\mathrm{X} 4$ in Plot (b) is linked to the apparent overestimation of the resistivity reconstruction in Plot (c).

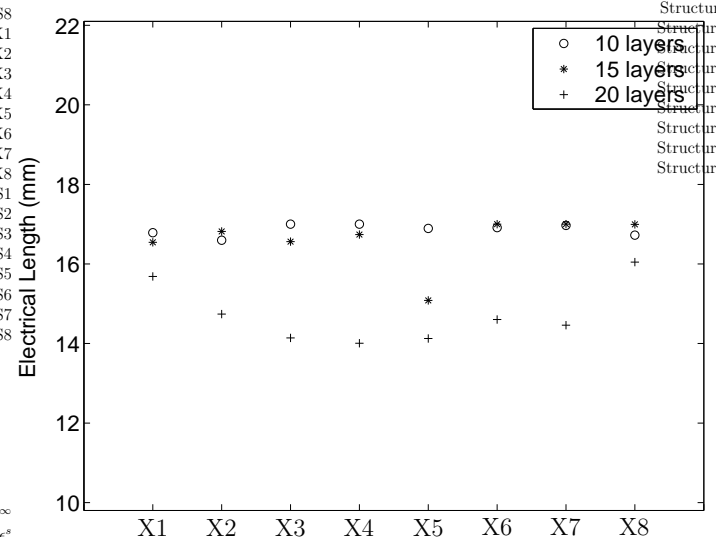

(a) Electrical Length Reconstruction.

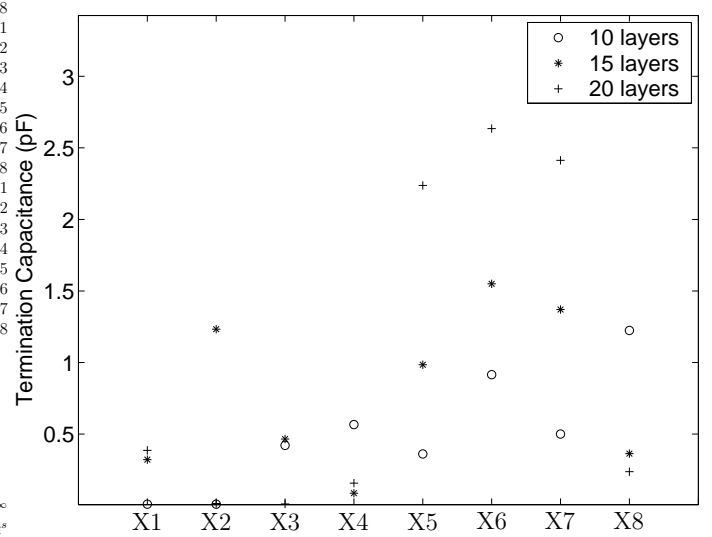

(b) Termination Capacitance Reconstruction.

Figure 16: Reconstructed Electrical Length and Termination Capacitance of X1 to X8: 10, 15 and 20 Layers.

The electrical length and termination capacitance were also reconstructed (see Figure 16). The electrical length of differing structures should be identical as this parameter is a property of the connector and not the structure. Plot (a) shows that for 10 and 15 layer reconstructions this is indeed the case with almost all reconstructions estimating $17 \mathrm{~mm}$. However at a 20 layer resolution the reconstructions are not constant but range from $14 \mathrm{~mm}$ to $16 \mathrm{~mm}$. Another cause for concern is that although the 10 and 15 layer reconstructions are consistently close to $17 \mathrm{~mm}$ this is greater than the expected value of $14 \mathrm{~mm}$. The electrical length is the only connector property which may be calculated from independent experiment and as such is thought to be the most accurate of all the estimates given in Table 1. Thus if the estimate is correct then the solver is overestimating 
the solution for 10 and 15 layer reconstructions and yielding inconsistent results for a 20 layer resolution.

In Figure 16, Plot (b) displays the reconstructions for the termination capacitance. The fact that differing structures yield different reconstructions is expected due to the dependence of the termination capacitance on the geometry. However different layer resolutions can produce noticeably different reconstructions for the same structure, similar to the simulated data case. This again suggests some interplay between the electrical parameters in the optimisation process (compare with Figure 10 Plot (b)).

\subsubsection{Electrical Reconstructions From Low to Mid Frequency Data}

In the case where low to mid frequency simulated data was used to reconstruct plate separations, it was found that the reconstructions were inferior to those found using the full frequency range simulated data (see Figure 7). Although not displayed, this is also true of plate separation reconstructions from experimental data. However it may not be true of reconstructions for electrical parameters. Indeed it may be that in this case the advantages of a more accurate model outweigh the disadvantages of reducing the amount of information available to the solver.

Reconstructions of resistivity and infinite and static permittivities for low to mid frequency data is considered (see Figure 17). Plot (a) again shows reconstructed infinite permittivities at physically feasible values. However on this occasion the values display less variance (than those of Figure 15) for different layers. Plot (b) also shows that by neglecting high frequency data the average value of the static permittivity has dropped to a more realistic value. Unfortunately Plot (c) reveals that the resistivity reconstructions are still diverging to the lower constraint and therefore to values which are unlikely to reflect the true resistivity.

Reconstruction of the electrical length and termination capacitance for low to mid frequency data is also considered (see Figure 18). Plot (a) reveals (with the exception of structure X8) reconstructions for the electrical length in the region of $14 \mathrm{~mm}$ for all layer resolutions. This agrees particularly well with the experimentally predicted value and it would therefore appear that use of low to mid frequency data has considerably improved the recovery of this parameter. The termination capacitance reconstructions in Plot (c), however, show little improvement beyond the fact that no reconstructions lie on the lower constraint. Once again reconstructions similar to those for the termination capacitance are also observed for the connector parameters. 


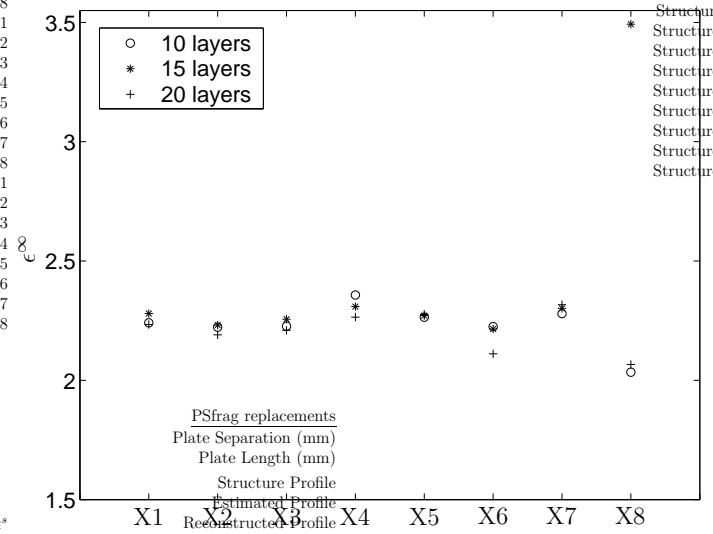

(a) Infinite Permittivity Reconstruction.

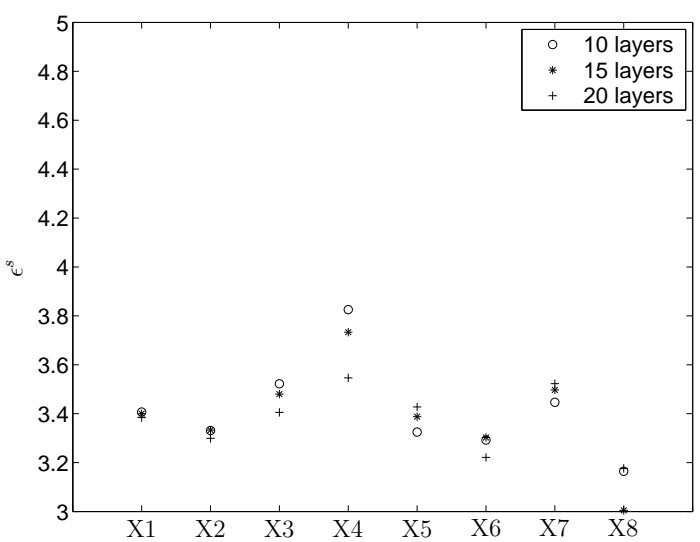

(b) Static Permittivity Reconstruction.

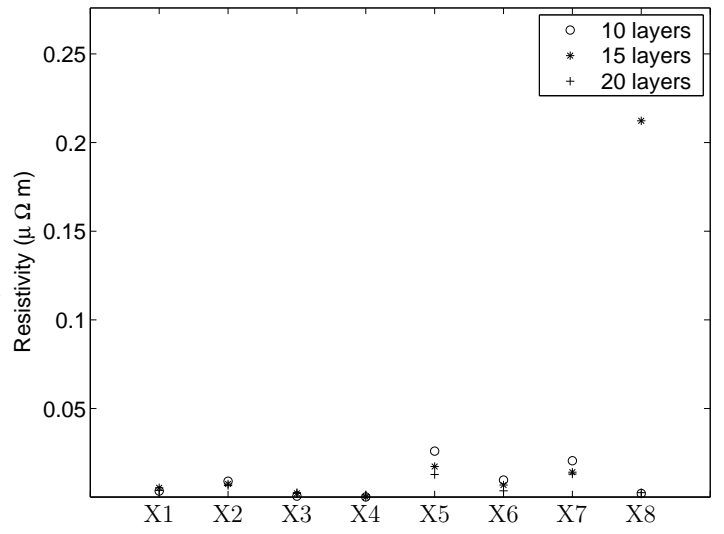

(c) Resistivity Reconstruction.

Figure 17: Reconstructed Structural Electrical Properties of X1 to X8: 10, 15 and 20 Layers (Low to Mid Frequency).

\section{Conclusions}

The results presented in this paper have demonstrated the effectiveness of this approach in recovering the electrical and geometrical properties of adhesively bonded test coupons. These properties were recovered using both experimental reflection coefficients and their simulated counterparts. Reconstructions of the plate separation were found to be in good qualitative agreement with experimental measurements. Quantitatively the reconstructions consistently underestimated the experimental measurements. It was found that increasing the width by $25 \%$ brought the reconstructions in line with the experimental measurements. However, such a large increase was difficult to justify and it was felt that the error in the true width was linked to an error in some electrical 


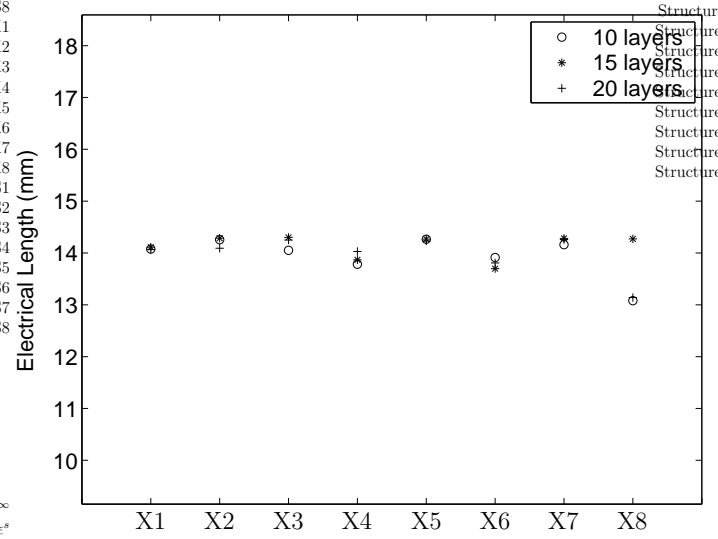

(a) Electrical Length

Reconstruction.

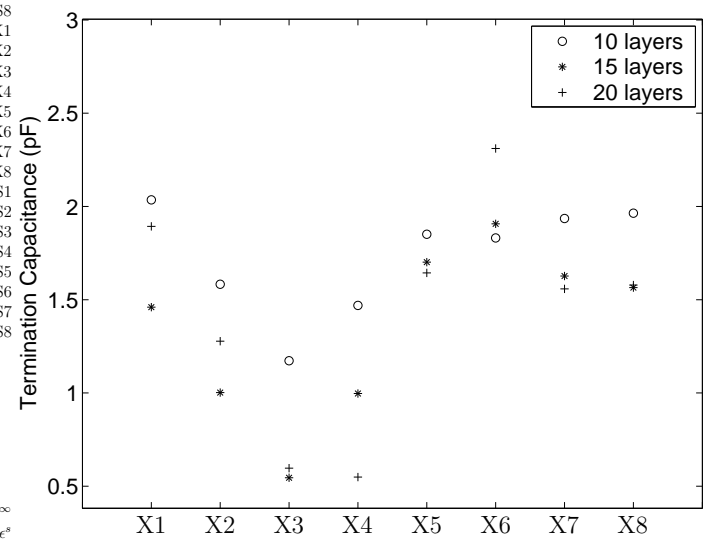

(b) Termination Capacitance Reconstruction.

Figure 18: Reconstructed Electrical Length and Termination Capacitance of X1 to X8: 10, 15 and 20 Layers.

parameters. From our results these are most likely to be the plate resistivity and the connector parameters. Higher resolution reconstructions were obtained that were qualitatively acceptable; however at resolutions beyond 20 layers the geometrical reconstructions became highly oscillatory.

Of the electrical parameters which were adequately recovered it was found that the accuracy could be improved by reducing the frequency range of the experimental data. This was to be expected as the model is based on low frequency approximations. However this reduction in the frequency range resulted in less accurate reconstructions of the plate separation.

Adhesively bonded structures are widely used in the aircraft industry and as such it is of paramount importance that a cost effective and efficient method of testing such structures is developed. The in-situ testing of such structures would amount to a significant improvement over current NDT methods in terms of both the cost and the time taken. This paper has presented a model and inverse method which, through further development, could be used to facilitate such in-situ testing.

\section{References}

[1] S. Affrossman, W. M. Banks, D. Hayward, and R. A. Pethrick, Non-destructive examination of adhesively bonded structures using dielectric techniques: review and some results, Proceedings Of The Institution Of Mechanical Engineers, 214 (2000), pp. 87-102. 
[2] W. Banks, P. Boinard, R. Crane, and R. Pethrick, Non destructive evaluation of adhesively bonded composite structures using high frequency dielectric spectroscopy, Journal of Material Science, 35 (2000), pp. 13311337 .

[3] W. Banks, F. Dumloin, S. Halliday, D. Hayward, Z. Li, and R. PETHRICK, Dielectric and mechanical assessment of water ingress into carbon fibre composite materials, Computers and Structures, 76 (2000), pp. $43-55$.

[4] W. Banks, F. Dumolin, D. Hayward, R. Pethrick, and Z. Li, Non-destructive examination of composite joint structures: a correlation of water absorbption and high-frequency dielectric propagation, Journal Of Physics D-Applied Physics, 29 (1996), pp. 233-239.

[5] K. Cole And R. Cole, Dispersion and absorption in dielctrics. I. alternating current characteristics, Journal Of Chemical Physics, 9 (1941), pp. 341-351.

[6] D. Davidson And R. Cole, Dielectric relaxation in glycerol, propylene glycol and n-propanol, Journal Of Chemical Physics, 19 (1951), pp. 14841490 .

[7] S. Havriliak AND S. Negami, A complex plane analysis of $\alpha$-dispersions in some polymer systems, Journal Of Polymer Science: Part C, 14 (1966), pp. $99-117$.

[8] D. Hayward and R. Pethrick, Dielectric non-destructive evaluation of adhesive bonded structures, tech. rep., Strathclyde University, 2001.

[9] S. B. Joshi, R. A. Pethrick, R. Gilmore, L. W. Yates, and D. HAYWARD, Environmental ageing of adhesively bonded joints. I. dielectric studies, Journal Of Adhesion, 62 (1997), pp. 281-315.

[10] S. B. Joshi, R. A. Pethrick, T. F. Gray, W. M. Banks, R. Gilmore, L. W. Yates, And D. HaYWARd, Environmental ageing of adhesively bonded joints. II. mechanical studies, Journal Of Adhesion, 62 (1997), pp. 317-335.

[11] C. Mackay, D. Hayward, A. J. Mulholland, S. McKee, and R. A. Pethrick, A Frequency Domain Inverse Problem for an Air Filled Line and Connector. (submitted).

[12] C. MACKAY, An Inverse Methodology for the Reconstruction of Spatial Dielectric Properties using Frequency Domain Reflection Coefficients., Ph.D. Thesis, University of Strathclyde, 2004. 
[13] Z. Li, S. Joshi, D. Hayward, R. Gilmore, and R. Pethrick, High frequency electrical measurements of adhesive bonded structures - an investigation of model parallel plate waveguide structures, NDT \& E International, 30 (1997), pp. 151-161.

[14] M. Nakhkash, Y. Huang, And M. FAng, Application of the multilevel single-linkage method to one-dimensional electromagnetic inverse scattering probelm, IEEE Transactions on Antennas and Propagation, 47 (1999), pp. $1658-1668$.

[15] M. Norgren And S. He, Optimal designs for nonuniform LCRG transmission lines, Journal of Electromagnetic Waves and Applications, 10 (1996), pp. 1113-1127.

[16] _ - An optimization approach to the frequency-domain inverse problem for a nonuniform, LCRG transmission line, IEEE Transactions On Microwave Theory And Techniques, 44 (1996), pp. 1503-1507.

[17] — A gradient-based optimization approach to the inverse problem for multi-layered structures, International Journal Of Applied Electromagnetics And Mechanics, 10 (1999), pp. 315-335.

[18] R. Pethrick, Comprehensive Composite Materials, vol. 5, Pergamon, Oxford and New York, 2000, ch. 15, pp. 360-390.

[19] S. Ramo, J. R. Whinnery, and T. Duzer, Fields and Waves in Communication Electronics, John Wiley, New York, 1994.

[20] D. A. Staelin, A. W. Morgenthaler, And J. A. Kong, Electromagnetic Waves, Prentice Hall, New Jersey, 1994. 


\section{A Analytical Jacobian Calculations}

For large-scale problems the computational cost can be substantially reduced if an analytical expression for the Jacobian $\mathbf{J} \in \mathbb{R}^{N \times 4 M}$ is employed. Passing the closed-form expression to the algorithm offers a greater degree of accuracy when obtaining the descent direction for the next iteration. More importantly, the algorithm's efficiency is greatly increased as the need to evaluate $N \times 4 M$ finite differences at each iteration is removed. Taking the derivative of (23) yields

$$
\begin{aligned}
\frac{\partial h_{k}}{\partial x_{i, j}}= & \frac{2}{\sqrt{N}} \Re\left\{\left(\overline{r_{c a l}^{+}\left(X ; \omega_{k}\right)-y^{+}\left(\omega_{k}\right)}\right) \frac{\partial}{\partial x_{i, j}} r_{c a l}^{+}\left(X ; \omega_{k}\right)\right. \\
& \left.+\left(\overline{r_{c a l}^{-}\left(X ; \omega_{k}\right)-y^{-}\left(\omega_{k}\right)}\right) \frac{\partial}{\partial x_{i, j}} r_{c a l}^{-}\left(X ; \omega_{k}\right)\right\} \\
= & \frac{2}{\sqrt{N}} \Re\left\{\left(\overline{r_{c a l, k}^{+}-y_{k}^{+}}\right) \frac{\partial r_{c a l, k}^{+}}{\partial x_{i, j}}+\left(\overline{r_{c a l, k}^{-}-y_{k}^{-}}\right) \frac{\partial r_{c a l, k}^{-}}{\partial x_{i, j}}\right\}
\end{aligned}
$$

where

$$
\begin{aligned}
& r_{c a l, k}^{+}=r_{c a l}^{+}\left(X ; \omega_{k}\right) \\
& r_{c a l, k}^{-}=r_{c a l}^{-}\left(X ; \omega_{k}\right)
\end{aligned}
$$

and where an overbar represents the complex conjugate. Note that (A. 1) is just an element from the $k^{\text {th }}$ row of the Jacobian corresponding to frequency number $k$; for clarity, the subscripts $k$ will be dropped from subsequent derivations. The analytical solution for layer $i$ is given by [11]

$$
r_{i}=\frac{r_{i+1}\left(P_{i}-Q_{i} E_{i}\right)+\left(E_{i}-1\right)}{r_{i+1}\left(1-E_{i}\right)+P_{i} E_{i}-Q_{i}} .
$$

This recursion relation may be differentiated with respect to the line parameter $x_{i, j}$ and the resultant recursion relation used to obtain the derivative $\frac{\partial r_{1}}{\partial x_{i, j}}$. The dielectric-filled lines were constructed in such a way that only the plate separation varied along the length. All the other parameters are assumed to be constant throughout the structure. In view of this

$$
\begin{aligned}
P_{i} & =P\left(\mathbf{u}, d_{i}\right) \\
Q_{i} & =Q\left(\mathbf{u}, d_{i}\right) \\
E_{i} & =E\left(\mathbf{u}, d_{i}\right)
\end{aligned}
$$

and

$$
r_{i+1}=r\left(\mathbf{u}, d_{i+1}, d_{i+2}, \ldots, d_{m}, \ldots, d_{M}\right)
$$

where $d_{i}$ is the plate separation of layer $i$ and $\mathbf{u}$ is the vector

$$
\mathbf{u}=\left[\epsilon^{s}, \epsilon^{\infty}, \rho\right]^{\mathrm{T}}
$$


containing the parameters which are layer independent. Therefore the line parameter vector for layer $i$ will be

$$
\mathbf{x}_{i}=\left[\mathbf{u}, d_{i}\right]
$$

and thus

$$
\left\{\mathbf{x}_{i}\right\}_{i=1}^{m-1} \bigcap\left\{\mathbf{x}_{i}\right\}_{i=m}^{M}=\mathbf{u}, \quad 1<m \leq M
$$

Proceeding with the differentiation for $(i+1) \leq m \leq M$ gives

$$
\frac{\partial r_{i}}{\partial x_{m, j}}=c_{i}^{(1)} \cdot \frac{\partial r_{i+1}}{\partial x_{m, j}}
$$

where

$$
c_{i}^{(1)}=\frac{E_{i}\left(P_{i}-Q_{i}\right)^{2}}{\left(r_{i+1}\left(1-E_{i}\right)+P_{i} E_{i}-Q_{i}\right)^{2}} .
$$

For the case $m \equiv i$ we obtain

$$
\frac{\partial r_{i}}{\partial u_{j}}=c_{i}^{(2)}\left\{c_{i}^{(3)} \frac{\partial P_{i}}{\partial u_{j}}+c_{i}^{(4)} \frac{\partial Q_{i}}{\partial u_{j}}+c_{i}^{(5)} \frac{\partial E_{i}}{\partial u_{j}}+c_{i}^{(7)} \frac{\partial r_{i+1}}{\partial u_{j}}\right\}
$$

where

$$
\begin{aligned}
c_{i}^{(2)} & =\frac{1}{\left(r_{i+1}\left(1-E_{i}\right)+P_{i} E_{i}-Q_{i}\right)^{2}} \\
c_{i}^{(3)} & =\left(1-E_{i}\right)\left(r_{i+1}-Q_{i}\right)^{2} \\
c_{i}^{(4)} & =E_{i}\left(E_{i}-1\right)\left(r_{i+1}-P_{i}\right)^{2} \\
c_{i}^{(5)} & =r_{i+1}^{2}\left(P_{i}-Q_{i}\right)+r_{i+1}\left(P_{i}^{2}-Q_{i}^{2}\right)+\left(P_{i}-Q_{i}\right) .
\end{aligned}
$$

and

$$
c_{i}^{(7)}=E_{i}\left(P_{i}-Q_{i}\right)^{2} .
$$

These recurrence relations are used in the same way as (A) to obtain the required set of gradients

$$
\left\{\frac{\partial r_{1}}{\partial x_{m, j}}\right\}_{m=1}^{M}
$$

for $j=1, \ldots, 4$; the derivative of the reflection coefficient $r_{1}$ with respect to the parameters in the $\mathrm{m}^{\text {th }}$ layer. This may be illustrated as follows; starting with $i \equiv m \equiv M$ use equation (A. 12) to obtain

$$
\frac{\partial r_{M}}{\partial x_{M, j}}=g\left(\mathbf{x}_{M}, r_{M+1}\right) .
$$


Repeated use of equation (A. 10) gives

$$
\begin{aligned}
& \frac{\partial r_{M-1}}{\partial x_{M, j}}=c_{M-1}^{(1)} \frac{\partial r_{M}}{\partial x_{M, j}}=c_{M-1}^{(1)}\left[g\left(\mathbf{x}_{M}, r_{M+1}\right)\right] \\
& \frac{\partial r_{M-2}}{\partial x_{M, j}}=c_{M-2}^{(1)} \frac{\partial r_{M-1}}{\partial x_{M, j}}=c_{M-2}^{(1)}\left[c_{M-1}^{(1)} g\left(\mathbf{x}_{M}, r_{M+1}\right)\right] \\
& \vdots \quad \vdots \quad \vdots \\
& \frac{\partial r_{i}}{\partial x_{M, j}}=c_{i}^{(1)} \frac{\partial r_{i+1}}{\partial x_{M, j}}=g\left(\mathbf{x}_{M}, r_{M+1}\right) \prod_{q=i}^{M-1} c_{q}^{(1)} \\
& \vdots \quad \vdots \quad \vdots \\
& \frac{\partial r_{1}}{\partial x_{M, j}}=c_{1}^{(1)} \frac{\partial r_{2}}{\partial x_{M, j}}=g\left(\mathbf{x}_{M}, r_{M+1}\right) \prod_{q=1}^{M-1} c_{q}^{(1)} .
\end{aligned}
$$

Similarly, beginning with $i \equiv m \equiv M-1$ use equation (A. 12) to obtain

$$
\frac{\partial r_{M-1}}{\partial x_{M-1, j}}=g\left(\mathbf{x}_{M-1}, r_{M}\right)
$$

and so (A. 10) yields

$$
\frac{\partial r_{1}}{\partial x_{M-1, j}}=c_{1}^{(1)} \frac{\partial r_{2}}{\partial x_{M-1, j}}=g\left(\mathbf{x}_{M-1}, r_{M}\right) \prod_{q=1}^{M-2} c_{q}^{(1)} .
$$

The general solution is

$$
\frac{\partial r_{1}}{\partial x_{i, j}}=c_{0}^{(1)} \frac{\partial r_{2}}{\partial x_{i, j}}=g\left(\mathbf{x}_{i}, r_{i+1}\right) \prod_{q=1}^{i-1} c_{q}^{(1)}
$$

where $0<i \leq M$. Note that $\frac{\partial r_{m<i}}{\partial x_{m, j}} \equiv 0$ as the reflection coefficient of any layer where $m<i$ has no dependence on $x_{m, j}$. Expressions for $\frac{\partial P_{i}}{\partial x_{i, j}}$, $\frac{\partial Q_{i}}{\partial x_{i, j}}$ and $\frac{\partial E_{i}}{\partial x_{i, j}}$ will now be derived. These expressions are in terms of $\frac{\partial a_{i}}{\partial x_{i, j}}, \frac{\partial b_{i}}{\partial x_{i, j}}$ and $\frac{\partial s_{i}}{\partial x_{i, j}}$ where

$$
\begin{aligned}
a_{i} & =\frac{1}{2} i \omega\left(\epsilon_{i} \frac{W}{d_{i}} Z_{0}+\frac{\mu_{i}}{Z_{0}} \frac{d_{i}}{W}\right) \\
b_{i} & =\frac{1}{2} i \omega\left(\epsilon_{i} \frac{W}{d_{i}} Z_{0}-\frac{\mu_{i}}{Z_{0}} \frac{d_{i}}{W}\right)
\end{aligned}
$$

and

$$
s_{i}=\sqrt{\epsilon_{i} \mu_{i}}
$$

The derivatives are

$$
\frac{\partial P_{i}}{\partial x_{i, j}}=-\left(\frac{\partial a_{i}}{\partial x_{i, j}}+i \omega \frac{\partial s_{i}}{\partial x_{i, j}}\right) \frac{1}{b_{i}}-\frac{\partial b_{i}}{\partial x_{i, j}}\left(-a+i \omega s_{i}\right) \frac{1}{b_{i}^{2}}
$$




$$
\frac{\partial Q_{i}}{\partial x_{i, j}}=-\left(\frac{\partial a_{i}}{\partial x_{i, j}}+i \omega \frac{\partial s_{i}}{\partial x_{i, j}}\right) \frac{1}{b_{i}}+\frac{\partial b_{i}}{\partial x_{i, j}}\left(a+i \omega s_{i}\right) \frac{1}{b_{i}^{2}}
$$

and

$$
\frac{\partial E_{i}}{\partial x_{i, j}}=-2 i \omega l_{i} \frac{\partial s_{i}}{\partial x_{i, j}} e^{\theta_{i}}
$$

where $\theta_{i}$ is given as

$$
\theta_{i}=-2 i \omega s_{i} l_{i}
$$

Note that $P_{i} Q_{i}=1$ also gives

$$
\frac{\partial Q_{i}}{\partial x_{i, j}}=-\frac{1}{P_{i}^{2}} \frac{\partial P_{i}}{\partial x_{i, j}}
$$

The derivatives $\frac{\partial s_{i}}{\partial x_{i, j}}, \frac{\partial a_{i}}{\partial x_{i, j}}$ and $\frac{\partial b_{i}}{\partial x_{i, j}}$ must now be calculated. These expressions are in terms of $\frac{\partial \epsilon_{i}}{\partial x_{i, j}}, \frac{\partial \mu_{i}}{\partial x_{i, j}}$ and $\frac{\partial g_{i}}{\partial x_{i, j}}$ where

$$
\begin{gathered}
\epsilon_{i}=\epsilon_{0}\left[\epsilon_{i}^{\infty}+\frac{\epsilon_{i}^{s}-\epsilon_{i}^{\infty}}{\left(1+(i \omega \tau)^{\alpha}\right)^{\beta}}\right] \\
\mu_{i}=\mu_{0}+\frac{(1-i)}{d_{i}} \sqrt{\frac{2 \mu_{0} \rho_{i}}{\omega}} .
\end{gathered}
$$

The derivatives are

$$
\begin{gathered}
\frac{\partial s_{i}}{\partial x_{i, j}}=\frac{1}{2}\left(\frac{\partial \epsilon_{i}}{\partial x_{i, j}} \sqrt{\frac{\mu_{i}}{\epsilon_{i}}}+\frac{\partial \mu_{i}}{\partial x_{i, j}} \sqrt{\frac{\epsilon_{i}}{\mu_{i}}}\right) \\
\frac{\partial a_{i}}{\partial x_{i, j}}=\frac{1}{2} i \omega\left[Z_{0} \frac{W}{d_{i}}\left(\frac{\partial \epsilon_{i}}{\partial x_{i, j}}-\epsilon_{i} \frac{1}{d_{i}} \frac{\partial d_{i}}{\partial x_{i, j}}\right)+\frac{1}{Z_{0}} \frac{d_{i}}{W}\left(\frac{\partial \mu_{i}}{\partial x_{i, j}}+\mu_{i} \frac{1}{d_{i}} \frac{\partial d_{i}}{\partial x_{i, j}}\right)\right]
\end{gathered}
$$

and

$$
\frac{\partial b_{i}}{\partial x_{i, j}}=\frac{1}{2} i \omega\left[Z_{0} \frac{W}{d_{i}}\left(\frac{\partial \epsilon_{i}}{\partial x_{i, j}}-\epsilon_{i} \frac{1}{d_{i}} \frac{\partial d_{i}}{\partial x_{i, j}}\right)-\frac{1}{Z_{0}} \frac{d_{i}}{W}\left(\mu_{i} \frac{1}{d_{i}} \frac{\partial d_{i}}{\partial x_{i, j}}+\frac{\partial \mu_{i}}{\partial x_{i, j}}\right)\right] .
$$

Expressions are now derived for $\frac{\partial \epsilon_{i}}{\partial x_{i, j}}, \frac{\partial \mu_{i}}{\partial x_{i, j}}$ and $\frac{\partial g_{i}}{\partial x_{i, j}}$

$$
\begin{gathered}
\frac{\partial \epsilon_{i}}{\partial \epsilon_{i}^{s}}=\frac{\epsilon_{0}}{\left(1+(i f \tau)^{\alpha}\right)^{\beta}} \\
\frac{\partial \epsilon_{i}}{\partial \epsilon_{i}^{\infty}}=\epsilon_{0}\left(1-\frac{1}{\left(1+(i f \tau)^{\alpha}\right)^{\beta}}\right) \\
\frac{\partial \mu_{i}}{\partial d_{i}}=\frac{i-1}{d_{i}^{2}} \pi \sqrt{\frac{2 \mu_{0} \rho_{i}}{\omega}}
\end{gathered}
$$




$$
\frac{\partial \mu_{i}}{\partial \rho_{i}}=\frac{(1-i)}{2 d_{i}} \pi \sqrt{\frac{2 \mu_{0}}{\rho_{i} \omega}} .
$$

In order for the full Jacobian to be supplied to the solver, derivatives with respect to $R, C_{1}, L_{c}, C_{2}$ and $C_{t}$ must be calculated. The reflection coefficient $r_{c a l}$ at the calibration plane is given by

$$
r_{c a l}=r_{0}(X) e^{-i \omega \frac{\Delta z}{c}}
$$

and so

$$
\frac{\partial r_{c a l}}{\partial x_{i, j}}=e^{-i \omega \frac{\Delta z}{c}} \frac{\partial r_{0}}{\partial x_{i, j}}, \quad 0 \leq i \leq M
$$

where now

$$
X=\left\{\mathbf{x}_{i}\right\}_{i=0}^{M+1}
$$

with $\mathbf{x}_{0}=\left[R, C_{1}, L_{c}, C_{2}\right]^{\mathrm{T}}$ and $\mathbf{x}_{M+1}=C_{t}$.

The reflection coefficient at $r_{0}$ is given by

$$
r_{0}=\frac{\left[\left(\omega^{2} C_{2} L_{c}-1\right) Z_{0}+i \omega L_{c}\right] Y_{1}\left(R, C_{1}, \bar{X}\right)+1-i \omega C_{2} Z_{0}}{\left[\left(\omega^{2} C_{2} L_{c}-1\right) Z_{0}-i \omega L_{c}\right] Y_{1}\left(R, C_{1}, \bar{X}\right)+1+i \omega C_{2} Z_{0}}
$$

where

$$
Y_{1}\left(R, C_{1}, \bar{X}\right)=\frac{1}{R+Z_{L}(\bar{X})}+i \omega C_{1}
$$

and

$$
\bar{X}=\left\{\mathbf{x}_{i}\right\}_{i=1}^{M+1} .
$$

Equation (A. 35) may be used to relate the derivative of $r_{0}$ to the that of $r_{c a l}$. Differentiating $r_{0}$ with respect to $C_{1}, R$ and $x_{i, j}(1<i<M+1)$ results in

$$
\left.\begin{array}{rl}
\frac{\partial r_{0}}{\partial C_{1}} & =c^{(6)} \frac{\partial Y_{1}}{\partial C_{1}} \\
\frac{\partial r_{0}}{\partial R} & =c^{(6)} \frac{\partial Y_{1}}{\partial R} \\
\frac{\partial r_{0}}{\partial x_{i, j}} & =c^{(6)} \frac{\partial Y_{1}}{\partial x_{i, j}}
\end{array}\right\}
$$

where

$$
c^{(6)}=-\frac{2 Z_{0}}{\left(\left[\left(\omega^{2} C_{2} L_{c}-1\right) Z_{0}-i \omega L_{c}\right] Y_{1}-\left(1+i \omega C_{2} Z_{0}\right)\right)^{2}} .
$$

Whereas differentiating with respect to $C_{2}$ and $L_{c}$ yields

$$
\frac{\partial r_{0}}{\partial C_{2}}=\left[\omega\left(\omega L_{c} Y_{1}-i\right)\left(1+i \omega L_{c} Y_{1}\right)\right] c^{(6)}
$$


and

$$
\frac{\partial r_{0}}{\partial L_{c}}=i \omega Y_{1} c^{(6)}
$$

The derivatives required in (A. 40) are found to be

$$
\begin{gathered}
\frac{\partial Y_{1}}{\partial C_{1}}=i \omega \\
\frac{\partial Y_{1}}{\partial R}=-\frac{1}{\left(R+Z_{L}(\bar{X})\right)^{2}}
\end{gathered}
$$

and

$$
\frac{\partial Y_{1}}{\partial x_{i, j}}=-\frac{1}{\left(R+Z_{L}(\bar{X})\right)^{2}} \frac{\partial Z_{L}}{\partial x_{i, j}} .
$$

Finally recall from (18) that

$$
Z_{L}(\bar{X})=\frac{1+r_{1}(\bar{X})}{1-r_{1}(\bar{X})} Z_{0}
$$

and so

$$
\frac{\partial Z_{L}}{\partial x_{i, j}}=\frac{2 Z_{0}}{\left(r_{1}(\bar{X})-1\right)^{2}} \frac{\partial r_{1}}{\partial x_{i, j}} .
$$

This is now used to obtain $\frac{\partial r_{1}}{\partial x_{m, j}}$; however the gradient for $C_{t}$ is first required.

Simple differentiation of equation (21) with respect to $C_{t}$ yields

$$
\frac{\partial r_{M+1}}{\partial C_{t}}=\frac{-2 i \omega Z_{0}}{\left(1+i \omega C_{t} Z_{0}\right)^{2}}
$$

Equation (A. 48) may be used in conjunction with the recurrence relation (A. 10) to obtain the derivative of $r_{0}$ with respect to $C_{t}$. 